\title{
PROP OF RIBBON HYPERGRAPHS AND STRONGLY HOMOTOPY INVOLUTIVE LIE BIALGEBRAS
}

\author{
SERGEI MERKULOV
}

\begin{abstract}
For any integer $d$ we introduce a prop $\mathcal{R H} r a_{d}$ of oriented ribbon hypergraphs (in which "edges" can connect more than two vertices) and prove that it admits a canonical morphism of props,$$
\mathcal{H} \text { olieb }_{d}^{\diamond} \longrightarrow \mathcal{R} \mathcal{H} r a_{d},
$$

$\mathcal{H}$ olieb $b_{d}^{\diamond}$ being the (degree shifted) minimal resolution of prop of involutive Lie bialgebras, which is non-trivial on every generator of $\mathcal{H}$ olieb $_{d}^{\diamond}$. We obtain two applications of this general construction.

First we show that for any graded vector space $W$ equipped with a family of cyclically (skew)symmetric higher products,

$$
\Theta_{n}:\left(\otimes^{n} W[d]\right)_{\mathbb{Z}_{n}} \longrightarrow \mathbb{K}[1+d], \quad n \geq 1,
$$

the associated vector space of cyclic words $C y c(W)=\oplus_{n \geq 0}\left(\otimes^{n} W\right)_{\mathbb{Z}_{n}}$ has a combinatorial $\mathcal{H}$ olieb $d_{d^{-}}$structure. As an illustration we construct for each natural number $N \geq 1$ an explicit combinatorial strongly homotopy involutive Lie bialgebra structure on the vector space of cyclic words in $N$ graded letters which extends the well-known Schedler's necklace Lie bialgebra structure from the formality theory of the Goldman-Turaev Lie bialgebra in genus zero.

Second, we introduced new (in general, non-trivial) operations in string topology. Given any closed connected and simply connected manifold $M$ of dimension $\geq 4$. We show that the reduced equivariant homology $\bar{H}_{\bullet}^{S^{1}}(L M)$ of the space $L M$ of free loops in $M$ carries a canonical representation of the dg prop Holieb $_{2-n}^{\diamond}$ on $\bar{H}_{\bullet}^{S^{1}}(L M)$ controlled by four ribbon hypergraphs explicitly shown in this paper.
\end{abstract}

Mathematics Subject Classifications (2000). 17B62, 18N70, 57Q10

KEY WORDS. Lie bialgebras, string topology, operads, props.

\section{Introduction}

1.1. Involutive Lie bialgebras. Lie bialgebras have been introduced by V. Drinfeld in [D1 in his studies of Yang-Baxter equations and the deformation theory of universal enveloping algebras. Nowadays involutive Lie bialgebras are used in many different areas of mathematics - in algebra, geometry, string topology, contact topology, theory of moduli spaces of algebraic curves, etc. (see, e.g., articles and books AKKN1, AKKN2, Ba1, CEG, CFL, Ch, CS, D1, D2, DCTT, ES, Ma, MW1, NW, T, S, as well as references cited there). The problem to prove Koszulness of the prop of involutive Lie bialgebras (and hence to understand their their homotopy theory) turned out to be a rather non-trivial one - it stayed open for almost a decade until its solution in [CMW]. It is a remarkable fact that the deformation theory of that prop is controlled by the mysterious Grothendieck-Teichmüller group MW2 which appears in many different areas of mathematics and explains, perhaps, the diversity of important mathematical problems which involve involutive Lie bialgebras.

The main result of this paper is an explicit construction of several families of strongly homotopy involutive Lie bialgebras using a new prop of ribbon hypergraphs $\mathcal{H G r a}, \forall d \in \mathbb{Z}$. We show two applications of our results - one is a purely combinatorial one generalizing a nice construction of T. Schedler in $[\underline{S}$, the second one deals with the beautiful theory of string topology introduced by M. Chas and D. Sullivan in [CS and introduces four new strongly homotopy operations on the reduced equivariant homology $\bar{H}_{\bullet}^{S^{1}}(L M)$ of the space of free loops in an arbitrary closed simply connected manifold $M$ of dimension $\geq 4$.

This work is much motivated by a particular class of involutive Lie bialgebra structures originating in the theory of punctured Riemann surfaces [G, T, AKKN1, AKKN2] and in the string topology of an arbitrary closed simply connected manifold [CS, CEG, CFL, NW]. 
Let $\widehat{\mathbb{K}}\left\langle\pi_{1}\left(\Sigma_{0, N+1}\right)\right\rangle$ stand for the completed group algebra of the fundamental group $\pi_{1}\left(\Sigma_{0, N+1}, \mathbb{K}\right)$ of the genus zero Riemann surface $\Sigma_{0, N+1}$ with $N+1$ boundary components, $N \geq 2$, and $H_{1}\left(\Sigma_{0, N+1}\right)$ for its first homology group over $\mathbb{K}$. Let

$$
\widehat{\mathfrak{g}}\left[\Sigma_{0, N+1}\right]:=\frac{\widehat{\mathbb{K}}\left\langle\pi_{1}\left(\Sigma_{0, N+1}\right)\right\rangle}{\left[\widehat{\mathbb{K}}\left\langle\pi_{1}\left(\Sigma_{0, N+1}\right), \widehat{\mathbb{K}}\left\langle\pi_{1}\left(\Sigma_{0, N+1}\right)\right\rangle\right]\right.}
$$

be the (completed) vector space spanned over a field $\mathbb{K}$ of characteristic zero by free homotopy classes of loops in $\Sigma_{0, N+1}$. Using intersections and self-intersection of loops Goldman and Turaev G, T] made this vector space into a filtered involutive Lie bialgebra1. Let

$$
\operatorname{gr} \widehat{\mathfrak{g}}\left[\Sigma_{0, N+1}\right]:=\frac{\widehat{\otimes^{\bullet}} H_{1}\left(\Sigma_{0, N+1}, \mathbb{K}\right)}{\left[\widehat{\otimes} H_{1}\left(\Sigma_{0, N+1}, \mathbb{K}\right), \widehat{\otimes} \cdot H_{1}\left(\Sigma_{0, N+1}, \mathbb{K}\right)\right]} \simeq C y c\left(W_{N}\right):=\prod_{n \geq 0}\left(\otimes^{n} W_{N}\right)_{\mathbb{Z}_{n}}
$$

be the associated graded involutive Lie bialgebra where

$$
W_{N}=\operatorname{span}_{\mathbb{K}}\left\langle x_{1}, \ldots x_{N}\right\rangle
$$

stands for the vector spaces generated by $N$ formal letters $x_{1}, \ldots, x_{N}$ (corresponding to the standard generators of $\left.H_{1}\left(\Sigma_{0, N+1}, \mathbb{K}\right)\right)$. The formality theorem AKKN1, AN, Ma establishes a highly non-trivial isomorphism of Lie bialgebras

$$
\widehat{\mathfrak{g}}\left[\Sigma_{0, N+1}\right] \longrightarrow \operatorname{gr} \widehat{\mathfrak{g}}\left[\Sigma_{0, N+1}\right]
$$

which depends on the choice of a Drinfeld associator. Thus the Goldman-Turaev Lie bialgebra structure can be understood in terms of its much simpler graded associated version which admits a purely combinatorial description. In fact, it admits two purely combinatorial descriptions. The first one is due to the general construction by Schedler [S] which associates to any quiver a so called necklace Lie bialgebra; the particular involutive Lie bialgebra structure on $\operatorname{gr} \widehat{\mathfrak{g}}\left[\Sigma_{0, N+1}\right] \simeq \operatorname{Cyc}\left(W_{N}\right)$ is the necklace one corresponding to the following quiver

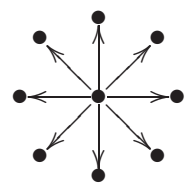

with $N$ legs. Put another way, for any natural number $N \geq 2$ T. Schedler's construction 2 gives us an involutive Lie bialgebra structure on $C y c\left(W_{N}\right)$ which admits a nice geometric interpretation.

The second combinatorial description of the necklace Lie bialgebra structure on $\operatorname{Cyc}\left(W_{N}\right)$ involves the $d=1$ case of a family of props of ribbon graphs $\mathcal{R G r a} a_{d}, d \in \mathbb{Z}$, which come equipped with canonical morphisms MW1

$$
\rho: \mathcal{L} i e b_{d}^{\diamond} \longrightarrow \mathcal{R G} \mathrm{Gr}_{d}
$$

from the prop of involutive Lie bialgebras; the map $\rho$ is non-trivial on both Lie and coLie generators of $\mathcal{L}_{i e b} b_{d}$ (which are assigned homological degree $1-d$ so that the case $d=1$ corresponds to the ordinary Lie bialgebras). It was shown in MW1 that for any $\mathbb{Z}$-graded vector space $V$ equipped with a pairing (which for $d=1$ is nothing but a skew-symmetric scalar product in $W$ )

$$
\Theta_{2}: \odot^{2}(W[d]) \quad \longrightarrow \mathbb{K}[1+d]
$$

there is an associated representation

$$
\rho_{\Theta_{2}}: \mathcal{R} \mathcal{G} r a_{d} \longrightarrow \mathcal{E} n d_{C y c(W)}
$$

\footnotetext{
${ }^{1}$ Strictly speaking this structure was originally defined modulo constant loops, i.e. on the quotient space $\widehat{\mathfrak{g}}\left[\Sigma_{0, N+1}\right] / \mathbb{K}$, but a choice of framing on $\Sigma_{0, N+1}$ permits us to extend that structure to the whole space. Put another way, the induced Lie bialgebra structure on $\widehat{\mathfrak{g}}\left[\Sigma_{0, N+1}\right]$ is canonical only on the quotient space $C y c^{\bullet}\left(W_{N}\right) / \mathbb{K} 1$, 1 being the empty cyclic word, and its extension to the whole space depends on some additional choices.

${ }^{2}$ It is worth emphasizing that Schedler's construction depends on the choice of a basis $\left(x_{1}, \ldots, x_{N}\right)$ in $W_{N}$, i.e. it depends essentially only on the natural number $N$.
} 
of the prop of ribbon graphs in the vector space

$$
C y c(W):=\prod_{n \geq 1}\left(\otimes^{n} W\right)_{\mathbb{Z}_{n}}
$$

spanned by cyclic words in elements of $W$, and hence there is an induced via the composition $\rho_{\Theta_{2}} \circ \rho$ an involutive Lie bialgebra structure in $C y c(W)$. The vector space $W_{N}$ has no natural pairings so we can not apply this construction immediately to get the necklace Lie bialgebra structure on $W_{N}$. However a certain "doubling" trick explained in $\$ \mathbf{2 . 5 . 1}$ does the job and gives us a canonical representation

$$
\rho_{N}: \mathcal{R G} \mathrm{Gr}_{1} \longrightarrow \mathcal{E} n d_{C y c\left(W_{N}\right)}
$$

which induces via the composition $\rho_{N} \circ \rho$ the required necklace Lie bialgebra structure on $\operatorname{Cyc}\left(W_{N}\right)$ (modulo terms depending on a particular choice of framing on $\Sigma_{0, N+1}$, i.e. both structures fully agree on the quotient space $C y c\left(W_{N}\right) / \mathbb{K} \mathbb{1}$, where $\mathbb{1}$ stands for the empty cyclic word; in fact, the particular map $\rho_{N}$ we construct in $\$ 2.5 .1$ corresponds to the blackboard framing as it was explained to the author by Yusuke Kuno $\mathrm{Ku}$.

Given any Poincare duality algebra $A=\mathbb{K} \oplus \bar{A}$ in degree $n$ (say, a one which models a closed simply connected $n$-dimensional manifold $M$ ), the associated space $W=\bar{A}^{*}[-1]$ comes equipped with a nondegenerate pairing $\Theta_{2}$ as above with $d=3-n$. Hence the above construction gives us a representation of $\mathcal{R G} r_{3-n}$ in $C y c(W)$ and hence induces a $\mathcal{L} i e b_{3-n}^{\diamond}$-algebra structure on that space. It has been proven in CEG] that $\mathcal{L} i e b_{3-n}^{\diamond}$-operations on $\operatorname{Cyc}\left(\bar{A}^{*}[-1]\right)$ respect the Hochschild differential $d_{H}$ and hence induce a $\mathcal{L} i e b_{3-n}^{\diamond}$-algebra structure on its cohomology $H^{\bullet}\left(C y c\left(\bar{A}^{*}[-1]\right), d_{H}\right)$. If $A$ happens to be a model of a closed simply connected $n$ manifold $M$, then there is an isomorphism of (co)homology group

$$
\bar{H}_{\bullet}^{S^{1}}(L M) \stackrel{\simeq}{\longrightarrow} H^{\bullet}\left(C y c\left(\bar{A}^{*}[-1]\right), d_{H}\right)
$$

where the l.h.s. stands for the reduced (negatively graded) equivariant cohomology of the space $L M$ of free loops in $M$. Hence $\bar{H}_{\bullet}^{S^{1}}(L M)$ comes equipped with a $\mathcal{L} i e b_{3-n}^{\diamond}$-algebra structure which was first discovered in a purely geometric way by M. Chas and D. Sullivan in [CS].

In this paper we introduce, for each integer $d \in \mathbb{Z}$, a prop of ribbon hypergraphs $\mathcal{H G}$ ra $a_{d}$ and show that there is a morphism of dg props

$$
\rho^{\diamond}: \mathcal{H} \text { olieb }_{d}^{\diamond} \longrightarrow \mathcal{H} \mathcal{G} r a_{d}
$$

which is non-trivial on every generator of the minimal resolution $\mathcal{H}$ olieb $b_{d}^{\diamond}$ of the prop $\mathcal{L}$ ieb $b_{d}^{\diamond}$. There is a natural commutative diagram

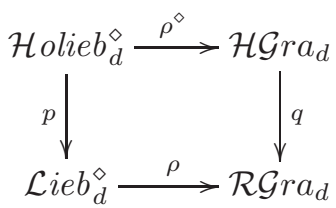

where the left vertical arrow $p$ is the canonical quasi-isomorphism and the right arrow $q$ is a "forgetful" map sending to zero all ribbon hypergraphs with at least one non-bivalent hyperedge.

Given any graded vector space $W$ equipped with cyclically (skew)invariant maps

$$
\Theta_{n}: \odot^{n}(W[d])_{\mathbb{Z}_{n}} \longrightarrow \mathbb{K}[1+d], \quad n \in \mathbb{N}_{\geq 1},
$$

there is a canonical representation

$$
\rho_{\Theta}: \mathcal{H} \mathcal{G} r a_{d} \longrightarrow \mathcal{E} n d_{C y c(W)}
$$

of the prop of ribbon hypergraphs, and hence a canonical strongly homotopy involutive Lie bialgebra structure

$$
\rho_{\Theta} \circ \rho^{\diamond}: \mathcal{H} \text { lieb }_{d}^{\diamond} \longrightarrow \mathcal{E} n d_{C y c(W)}
$$

on the space of cyclic words $C y c(W)$ in letters from $W$. If all $\Theta_{n}$ vanish except for $n=2$ we recover the previous result from MW1.

We consider two applications of the above construction, one is in purely algebra and another one is in string topology. 
Using multi-tuple generalization of the "doubling" trick used in the construction of representation (2) we introduce an explicit highly non-trivial strongly homotopy involutive Lie bialgebra structure,

$$
\hat{\rho}_{N}: \mathcal{H} \text { olieb }{ }_{1}^{\diamond} \mathcal{E} d_{C y c\left(\widehat{W}_{N}\right)}
$$

in the vector space generated by cyclic words in $\mathbb{Z}$-graded letters $\left\{x_{1}[-p], \ldots, x_{N}[-p]\right\}_{p \in \mathbb{N}}$, where $x_{i}[-p]$ stands for the formal letter $x_{i}$ to which we assigned homological degree $p$. When all letters are concentrated in degree zero, one recovers Schedler's necklace Lie bialgebra associated to the quiver (1).

Let $M$ be an arbitrary closed simply connected $n$-dimensional manifold, and $\Omega_{M}^{\bullet}$ its de Rham algebra. Using the integration morphism (more precisely, its version in a Poincare duality algebra modelling $M$ )

$$
\begin{array}{ccc}
\otimes^{3} \Omega_{M}^{\bullet} & \longrightarrow & \mathbb{R} \\
\alpha \otimes \beta \otimes \gamma & \longrightarrow & \int_{M} \alpha \wedge \beta \wedge \gamma
\end{array}
$$

we prove that the reduced equivariant cohomology $\bar{H}_{\bullet}^{S^{1}}(L M)$ of the space $L M$ of free loops in $M$ comes equipped canonically with a $\mathcal{H}$ olie $b_{2-n}^{\diamond}$-structure whose only possibly non-trivial operations are controlled by the following four generators of $\mathcal{H} o l i e b_{2-n}^{\diamond}$ (see $\S 2$ for their definition),

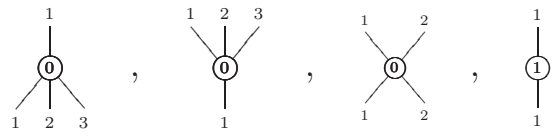

which in turn are respectively controlled via the above mentioned morphism $\rho^{\diamond}$ by the following ribbon hypergraphs from $\mathcal{R} \mathcal{H} \mathrm{ra}_{2-n}$,

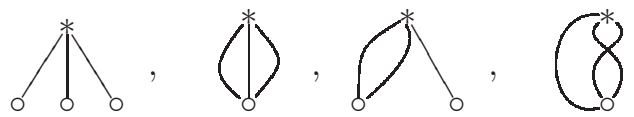

with vertices and boundaries appropriately (skew)symmetrized (so that their labelling is omitted). It is easy to see that these four new $\mathcal{H}$ olieb ${ }_{2-n}^{\diamond}$ operations on $\bar{H}_{\bullet}^{1}(L M)$ are non-trivial for $M=\mathbb{C P}^{n}$ with $n \geq 3$.

1.2. Structure of the paper. In $\S 2$ we remind basic facts about the prop of (degree shifted) involutive Lie bialgebras $\mathcal{L} i e b_{d}^{\diamond}$ and its minimal resolution $\mathcal{H}_{o l i e} b_{d}^{\diamond}$, and their interrelations with $\mathcal{H} o \mathcal{B} \mathcal{V}_{d}^{\text {com }}$-algebras (see CMW for more details and proofs). In $\S 3$ we describe in detail the prop of ribbon hypergraphs which is a natural extension of the prop of ribbon graphs introduced in MW1, and then construct its canonical representation in the space of cyclic words $C y c(W)$ associated with any graded vector space $W$ equipped with a family of cyclically (skew)symmetric higher products (which is a rather straightforward generalization of the similar construction in [MW1]). In $\S 4$ we explain the main (and not that straightforward) result of this paper - a construction of an explicit morphism of props, $\mathcal{H}$ olieb ${ }_{d}^{\diamond} \rightarrow \mathcal{R} \mathcal{H} r a_{d}$, which is non-trivial on every generator of $\mathcal{H}$ olieb $b_{d}^{\diamond}$ This result gives us a large family of explicit strongly homotopy involutive Lie bialgebras; in particular, for any natural number $N \in \mathbb{N}_{\geq 1}$ we show in $\S 5$ an explicit strongly homotopy involutive Lie bialgebra structure on the vector space of cyclic words in $\mathbb{Z}$-graded formal letters which extends the well-known Schedler's necklace Lie bialgebra structure $[\underline{S}$ from the formality theory of the GoldmanTuraev Lie bialgebra in genus zero. In $\S 6$ we discuss an application of the main construction to string topology.

1.3. Some notation. The set $\{1,2, \ldots, n\}$ is abbreviated to $[n]$; its group of automorphisms is denoted by $\mathbb{S}_{n}$. The trivial (resp., sign) one-dimensional representation of $\mathbb{S}_{n}$ is denoted by $\mathbb{1}_{n}$ (resp., sgn $n_{n}$ ). The cardinality of a finite set $A$ is denoted by $\# A$. If $V=\oplus_{i \in \mathbb{Z}} V^{i}$ is a graded vector space, then $V[n]$ stands for the graded vector space with $V[n]^{i}:=V^{i+n}$; for $v \in V^{i}$ we set $|v|:=i$. The canonical degree -1 isomorphism $V \rightarrow V[1]$ is denoted by $\mathfrak{s}$; one has $\left|\mathfrak{s}^{k} v\right|=|v|-k$ for any homogeneous element $v \in V$.

For a $\operatorname{prop}($ erad $) \mathcal{P}$ we denote by $\mathcal{P}\{n\}$ a prop(erad) which is uniquely defined by the following property: for any graded vector space $W$ a representation of $\mathcal{P}\{n\}$ in $W$ is identical to a representation of $\mathcal{P}$ in $W[n]$. For a module $V$ over a group $G$ we denote by $V_{G}$ the vector space of coinvariants: $V /\{g(v)-v \mid v \in V, g \in G\}$ and by $V^{G}$ the subspace of invariants: $\{\forall g \in G: g(v)=v, v \in V\}$. We always work over a field $\mathbb{K}$ of characteristic zero so that if $G$ is finite, then these spaces are canonically isomorphic, $V_{G} \cong V^{G}$. 
Acknowledgement. It is a pleasure to thank Anton Alekseev, Martin Kassabov, Nariya Kawazumi, Anton Khoroshkin, Yusuke Kuno, Florian Naef and Thomas Willwacher for valuable discussions. This text is based partially on the talk given by the author at the workshop "Poisson geometry of moduli spaces, associators and quantum field theory" organized at the Simons Center of Geometry and Physics in June 2018. Most of this text was also written there, and the author acknowledges with thanks the excellent working conditions at the SCGP.

\section{Strongly homotopy involutive Lie bialgebras as commutative $B V$ algebras and vice versa}

\subsection{Reminder on the prop of strongly homotopy (involutive) Lie bialgebras [CMW]. Let}

$$
\mathcal{L} \text { ieb }_{d}:=\mathcal{F} \text { ree }\langle E\rangle /\langle\mathcal{R}\rangle,
$$

be the quotient of the free prop(erad) generated by an $\mathbb{S}$-bimodule $E=\{E(m, n)\}_{m, n \geq 1}$ with

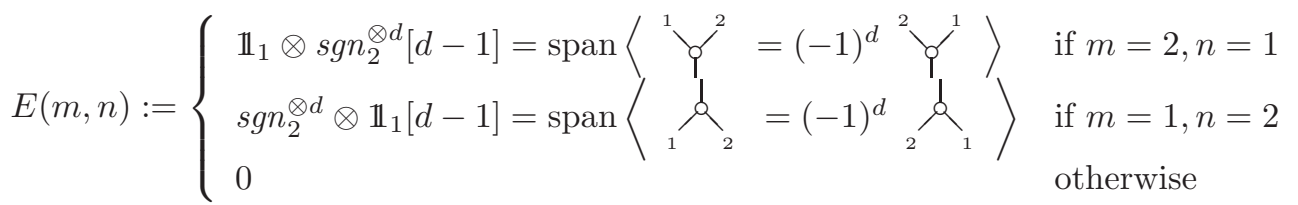

by the ideal $\langle\mathcal{R}\rangle$ generated by the following relations

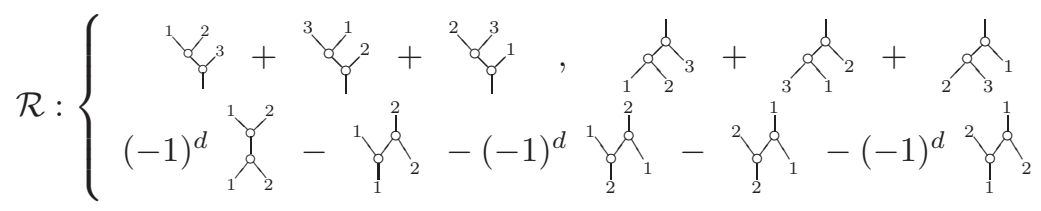

It is called the prop of (degree shifted) Lie bialgebras.

The prop of involutive Lie $d$-bialgebras is defined similarly,

$$
\mathcal{L} i e b_{d}^{\diamond}:=\mathcal{F} r e e\langle E\rangle /\left\langle\mathcal{R}_{\diamond}\right\rangle
$$

but with a larger set of relations,

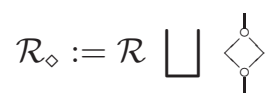

A representation $\rho:{\mathcal{L} i e b_{d}}_{\rightarrow} \mathcal{E} n d_{V}$ (resp., $\rho: \mathcal{L} i e b_{d}^{\diamond} \rightarrow \mathcal{E} n d_{V}$ ) in a graded vector space $V$ provides the latter with two operations

$$
[,]:=\rho(\mathcal{R}): \odot^{2}(V[d]) \rightarrow V[1+d], \quad \Delta:=\left(\wp^{\prime}\right): V[d] \longrightarrow \odot^{2}(V[d])[1-2 d]
$$

which satisfy the compatibility conditions controlled by the relations $\mathcal{R}$ (resp. $\mathcal{R}^{\diamond}$ ). If $d=1$, it is precisely the prop of ordinary involutive Lie bialgebras and is often denoted by $\mathcal{L} i e b^{\diamond}$.

The properads behind the props $\mathcal{L}_{i e b_{d}}$ and $\mathcal{L} i e b_{d}^{\diamond}$ are Koszul so that their minimal resolutions, $\mathcal{H}$ olieb $b_{d}$ and respectively $\mathcal{H}$ olieb $_{d}^{\diamond}$, are relatively "small" (see [CMW, MaVo, V] and references cited there). The dg prop $\mathcal{H}$ olieb $_{d}$ is generated by the (skew)symmetric corollas of homological degree $1-d(m+n-2)$

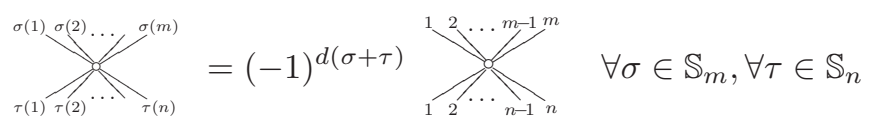

The differential is given on the generators by

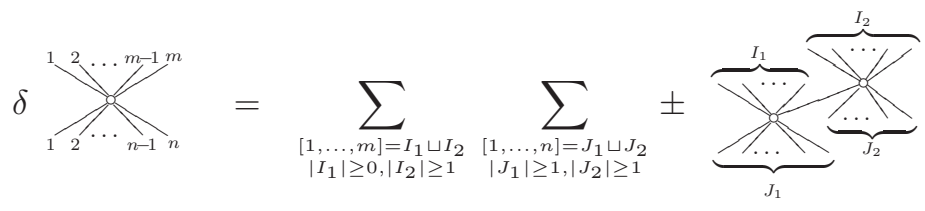


where the signs on the r.h.s are uniquely fixed by the fact that they all equal to +1 for $d$ odd. On the other hand, the dg prop $\mathcal{H}$ olieb $_{d}^{\diamond}$ is generated by the (skew)symmetric corollas of degree $1-d(m+n+2 a-2)$,

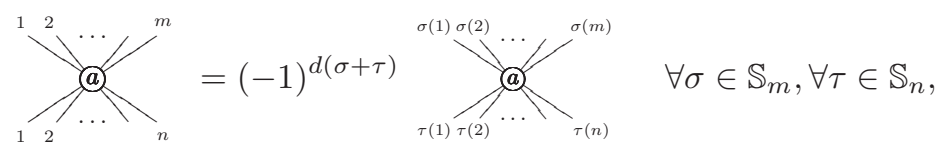

where $m+n+a \geq 3, m \geq 1, n \geq 1, a \geq 0$; the differential is given by

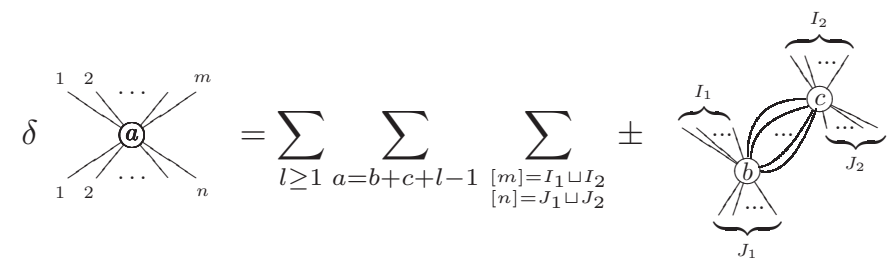

where the summation parameter $l$ counts the number of internal edges connecting the two vertices on the r.h.s., and the signs are fixed by the fact that they all equal to +1 for $d$ even. If $d=1$, it is called the prop of strongly homotopy involutive Lie bialgebras and is denoted by $\mathcal{H}$ olieb ${ }^{\diamond}$.

There is a canonical injection of props $\mathcal{H}$ olieb $_{d} \rightarrow \mathcal{H}$ olieb $_{d}^{\diamond}$ sending a generator $\mathcal{H}$ lieb $_{d}$ into the corresponding generator of $\mathcal{H}$ olieb ${ }_{d}^{\diamond}$ with $a=0$.

Sometimes it is more suitable to work with the degree shifted version $\mathcal{H}$ olieb $b_{d}^{\diamond}\{d\}$ of the prop $\mathcal{H}$ olieb $b_{d}^{\diamond}$ wich is defined uniquely by the following property: a representation of $\mathcal{H} o l i e b_{d}^{\diamond}\{d\}$ in a vector space $V$ is identical to the representation of $\mathcal{H}$ olieb $b_{d}^{\diamond}$ in $V[d]$. The prop $\mathcal{H}$ olieb $b_{d}^{\diamond}\{d\}$ is generated by the symmetric corollas of degree $1-2 d(n+a-1)$,

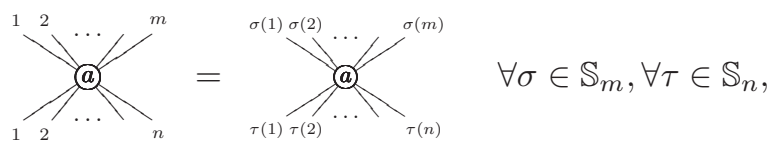

The differential is given by the above formula with the slightly ambiguous symbol \pm replaced by +1 (i.e. omitted); hence the sign rules become especially simple in this case.

2.2. Holieb $_{d}^{\diamond}$-algebras as Maurer-Cartan elements [CMW]. According to the general theory [MV], the set of $\mathcal{H}$ olieb $_{d}^{\diamond}\{d\}$-algebra structures in a dg vector space $(V, \delta)$ can be identified with the set of MaurerCartan elements of a graded Lie algebra,

$$
\mathfrak{g}_{V}^{\diamond}:=\operatorname{Hom}(V, V)[1] \oplus \operatorname{Def}\left(\mathcal{H} \text { olieb } b_{d}^{\diamond}\{d\} \stackrel{0}{\rightarrow} \mathcal{E} n d_{V}\right),
$$

controlling deformations of the trivial morphism which sends all the generators of $\mathcal{H}$ olieb ${ }_{d}^{\diamond}\{d\}$ to zero in $\mathcal{E} n d_{V}$. The summand $\operatorname{Hom}(V, V)[1]$ takes care about deformations of the given differential $\delta$ in $V$. Using the explicit description of the dg prop $\mathcal{H} o l i e b_{d}^{\diamond}\{d\}$ given at the end of the previous subsection, one can identify $\mathfrak{g}_{V}^{\diamond}$ as a $\mathbb{Z}$-graded vector space with

$$
\begin{aligned}
\mathfrak{g}_{V}^{\diamond} & =\prod_{a \geq 0, m, n \geq 1} \operatorname{Hom}_{\mathbb{S}_{m} \times \mathbb{S}_{n}}\left(\operatorname{Id}_{m} \otimes \operatorname{Id}_{n}[2 d(n+a-1)-1], \operatorname{Hom}\left(V^{\otimes n}, V^{\otimes m}\right)\right)[-1] \\
& =\prod_{a \geq 0, m, n \geq 1} \operatorname{Hom}\left((V[2 d])^{\odot n}, V^{\odot m}\right)[-2 d a+2 d]
\end{aligned}
$$

Assume $V$ has a countable basis $\left(x_{1}, x_{2}, \ldots\right)$, and let $\left(p^{1}, p^{2}, \ldots\right)$ stand for the associated set of dual generators of $\operatorname{Hom}(V[2 d], \mathbb{K})$ (with $\left|p^{i}\right|+\left|x_{i}\right|=2 d$ ), then the degree shifted vector space $\mathfrak{g}_{V}^{\diamond}[-2 d]$ can be identified with the subspace of a graded commutative ring

$$
\mathfrak{g}_{V}^{\diamond}[-2 d] \subset \mathbb{K}\left[\left[x^{i}, p_{i}, \hbar\right]\right]
$$

spanned by those formal power series $f(x, p, \hbar)$ which satisfy the conditions

$$
\left.f(x, p, \hbar)\right|_{x_{i}=0}=0,\left.\quad f(x, p, \hbar)\right|_{p^{i}=0}=0
$$


i.e. which belong to the maximal ideal generated by the products $x_{i} p^{j}$. Here $\hbar$ is a formal parameter ${ }^{3}$ of degree $2 d$. The algebra $\mathbb{K}\left[\left[x^{i}, p_{i}, \hbar\right]\right]$ has a classical associative star product given explicitly (up to standard Koszul signs) as follows

$$
f *_{\hbar} g:=\sum_{k=0}^{\infty} \frac{\hbar^{k}}{k !} \sum_{i_{1}, \ldots, i_{k}} \pm \frac{\partial^{k} f}{\partial p^{i_{1}} \cdots \partial p^{i_{k}}} \frac{\partial^{k} g}{\partial x_{i_{1}} \cdots \partial x_{i_{k}}}
$$

The Lie brackets in the degree shifted deformation complex $\mathfrak{g}_{V}^{\diamond}[-2 d]$ are then given by [DCTT]

$$
[f, g]=\frac{f * \hbar g-(-1)^{|f||g|} g * \hbar f}{\hbar}
$$

Hence $\mathcal{H}$ olieb $_{d}^{\diamond}\{d\}$-algebra structures in a graded vector space $V$ (with a countable basis) are in 1-1 correspondence with homogeneous formal power series $\Gamma \in \mathfrak{g}_{V}^{\diamond}[-2 d]$ of degree $1+2 d$ satisfying the equation

$$
\Gamma *_{\hbar} \Gamma=\sum_{k=1}^{\infty} \frac{\hbar^{k-1}}{k !} \sum_{i_{1}, \ldots, i_{k}} \pm \frac{\partial^{k} \Gamma}{\partial p^{i_{1}} \cdots p^{i_{k}}} \frac{\partial^{k} \Gamma}{\partial x_{i_{1}} \cdots \partial x_{i_{k}}}=0,
$$

This compact description of all higher homotopy involutive Lie bialgebra operations in $V$ is quite useful in making an explicit link between $\mathcal{H}$ olieb $_{d}^{\diamond}$ algebras and commutative $B V$-algebras which is outlined next.

2.3. Commutative Batalin-Vilkovisky $d$-algebras. A commutative Batalin-Vilkovisky d-algebra or, shortly, a $\mathcal{H} o \mathcal{B V}_{d}^{\text {com }}$-algebra is, by definition $\underline{\mathrm{Kr}}$, a differential graded commutative algebra $(V, \delta)$ equipped with a countable collections of homogeneous linear maps, $\left\{\Delta_{a}: V \rightarrow V,\left|\Delta_{a}\right|=1-2 d a\right\}_{a \geq 1}$, such that each operator $\Delta_{a}$ is of order $\leq a+1$ (with respect to the given multiplication) and the equations,

$$
\sum_{a=0}^{n} \Delta_{a} \circ \Delta_{n-a}=0, \quad \text { with } \Delta_{0}:=-\delta
$$

hold for any $n \in \mathbb{N}$. These equations are equivalent to one equation,

$$
\Delta_{\hbar}^{2}=0
$$

for the formal power series of operators

$$
\Delta_{\hbar}:=\sum_{a=0}^{\infty} \hbar^{a} \Delta_{a},
$$

where the formal power variable $\hbar$ is assigned degree $2 d$. Let us denote by $\mathcal{H} o \mathcal{B} \mathcal{V}_{d}^{\text {com }}$ the $\operatorname{dg}$ operad governing commutative $B V d$-algebras. This operad is the quotient of the free operad generated by one binary operation of degree zero $\Omega_{2}=\lambda_{1}$ (standing for graded commutative multiplication) and a countable family of unary operations $\left\{\begin{array}{l}\mid \\ a\end{array}\right\}_{a \geq 1}$ of homological degree $1-2 d a$ modulo the ideal $I$ generated by the standard associativity relation for $\underset{d}{d}$ and the compatibility relations involving the latter and the unary operations which assure that each unary operation $\underset{a}{a}$ is of order $\leq a+1$ with respect to the multiplication. The differential $\delta$ in $\mathcal{B} \mathcal{V}_{\infty}^{\text {com }}$ is given by

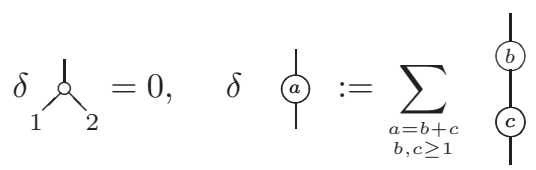

Let $J$ be the differential closure of an ideal in $\mathcal{H}_{o} \mathcal{B} \mathcal{V}_{d}^{c o m}$ generated by operations $\prod_{a}^{1}$ with $a \geq 2$. The quotient $\mathcal{H} \circ \mathcal{B} \mathcal{V}_{d}^{\text {com }} / J$ is precisely of the operad of (degree shifted) Batalin-Vilkovisky algebras $\mathcal{B} \mathcal{V}_{d}$. It was proven in [CMW] that the canonical projection $\mathcal{H} \mathcal{B V}_{d}^{\text {com }} \longrightarrow \mathcal{B} \mathcal{V}_{d}$ is quasi-isomorphism of operads.

\footnotetext{
${ }^{3}$ For a vector space $W$ we denote by $W[[\hbar]]$ the vector space of formal power series in $\hbar$ with coefficients in $W$. For later use we denote by $\hbar^{m} W[[\hbar]]$ the subspace of $W[[\hbar]]$ spanned by series of the form $\hbar^{m} f$ for some $f \in W[[\hbar]]$.
} 
2.4. From $\mathcal{H} o l i e b_{d}^{\diamond}$-algebras to $\mathcal{H} o \mathcal{B} \mathcal{V}_{d}^{\text {com }}$-algebras and back. Recall that a $\mathcal{H} o l i e b_{d}^{\diamond}\{d\}$-algebra structure in a graded vector space $V$ (i.e. a $\mathcal{H}$ olieb $_{d}^{\diamond}$ structure in $V[d]$ ) can be identified with a degree $1+2 d$ element $\Gamma$ in $\mathfrak{g}_{V}^{\diamond}[-2 d] \subset \mathbb{K}\left[\left[p^{i}, x_{i}, \hbar\right]\right]$ satisfying equation (8). Out of this datum one creates a $\mathcal{H}_{o} \mathcal{B V}_{d}^{c o m}$ algebra structure on $\widehat{\odot}^{\bullet} V$ (the completed symmetric tensor algebra on $V$ ), i.e. a representation

$$
\rho: \mathcal{H} o \mathcal{B V}_{d}^{\text {com }} \longrightarrow \mathcal{E}_{n} d_{\widehat{\odot}(V)},
$$

which is given explicitly as follows [CMW],

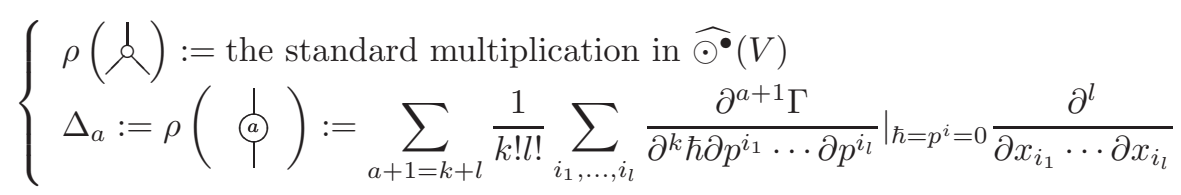

This explicit correspondence can be equivalently understood as a morphism of dg operads

$$
F: \mathcal{H o B V}_{d}^{\text {com }} \longrightarrow \mathcal{O}\left(\mathcal{H}_{\text {olieb }}^{\diamond}\{d\}\right)
$$

given explicitly on generators as follows,

$$
\begin{aligned}
& F\left(\begin{array}{l}
\ell_{2} \\
1
\end{array}\right):=\text { (1) (2) } \\
& F\left(\begin{array}{c}
1 \\
1
\end{array}\right)=\sum_{m \geq 1} \sum_{a+1=p+q}\left(\begin{array}{c}
\left(\begin{array}{c}
a \\
\cdots \\
k \\
\cdots \\
i
\end{array}\right) \\
i
\end{array}\right)
\end{aligned}
$$

where

$$
\begin{aligned}
& \mathcal{O}: \text { Category of props } \longrightarrow \text { Category of operads } \\
& \mathcal{P} \quad \longrightarrow \quad \mathcal{O}(\mathcal{P})
\end{aligned}
$$

is the polydifferential functor introduced in [MW1] (we refer to $§ 5.1$ of [MW1] or $§ 2.2$ of MW2 for full details explaining, in particular, the symbols on the r.h.s. of the above formula; these sections can be read independently of the rest of both papers). Its main defining property is that, given any representation $\rho: \mathcal{P} \rightarrow \mathcal{E} n d_{V}$ of a prop $\mathcal{P}$ in a graded vector space $X$, there is an associated representation $\mathcal{O}(\rho): \mathcal{O}(\mathcal{P}) \rightarrow$ $\mathcal{E} n d_{\overparen{\odot} \times}$ of the operad $\mathcal{O}(\mathcal{P})$ in the completed free graded commutative algebra $\widehat{\odot} X$ such that elements of $\mathcal{P}$ acts on $\widehat{\odot}(X)$ as polydifferential operators. The symbol on the r.h.s. of (11) is precisely the polydifferential operator corresponding to the generator

$$
\rho: \mathcal{H} o \mathcal{B V}_{d}^{c o m} \longrightarrow \mathcal{E} n d_{\widehat{\odot}(V)},
$$

such that

$$
\rho(\boldsymbol{l}):=\text { the standard multiplication in } \widehat{\odot}(V)
$$

it was proven in DCTT that it factors through the composition

$$
\rho: \mathcal{H}_{o} \mathcal{B V}_{d}^{\text {com }} \stackrel{F}{\longrightarrow} \mathcal{O}\left(\mathcal{H}_{\text {olieb }}^{\diamond}\{d\}\right) \stackrel{\mathcal{O}\left(\rho^{\prime}\right)}{\longrightarrow} \mathcal{O}\left(\mathcal{E} n d_{V}\right)=\mathcal{E} n d_{\overparen{\odot} V}
$$

for some representation $\rho^{\prime}: \mathcal{H}$ olieb $^{\diamond}\{d\} \rightarrow \mathcal{E} n d_{V}$. Put another way, one can read all the higher homotopy

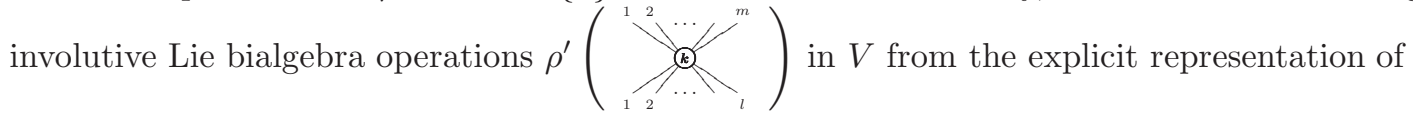

$$
\rho\left(\mathfrak{a}^{1}\right)=\sum_{m \geq 1} \frac{1}{m !} \sum_{a+1=k+l} \sum_{i_{\bullet}, j} C^{(k) j_{i_{1} \ldots j_{l}}} x_{j_{1}} \ldots x_{j_{m}} \frac{\partial^{l}}{\partial x_{i_{1}} \cdots \partial x_{i_{l}}}
$$


of the generator $@$ as a differential operator of order $\leq a+1$ on $\widehat{\odot} \cdot V \mathbb{K}\left[\left[x_{i}\right]\right]$ : the linear map

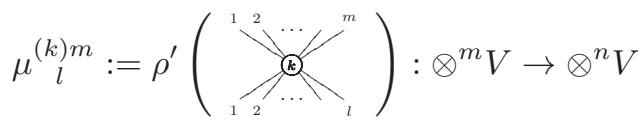

is given in the basis $\left\{x_{i}\right\}$ by (modulo the standard Koszul signs) by

$$
\mu_{l}^{(k) m}\left(x_{i_{1}} \otimes \ldots \otimes x_{i_{l}}\right)=\sum_{j_{\bullet}} C^{(k) j_{1} \ldots j_{l}} x_{j_{1}} \otimes \ldots \otimes x_{j_{m}}
$$

We shall use this one-to-one correspondence heavily in the proof of the Main Theorem 4.3 below.

2.5. A basic example of an involutive Lie bialgebra. Let $W$ be a graded vector space equipped with a linear map $\Theta: \odot^{2}(W[d]) \rightarrow \mathbb{K}[1+d]$ for some $d \in \mathbb{Z}$. This map is the same as a degree $1-d$ pairing $\Theta: W \otimes W \rightarrow \mathbb{K}[1-d]$ satisfying the (skew) symmetry condition,

$$
\Theta\left(w_{1}, w_{2}\right)=(-1)^{d+\left|w_{1}\right|\left|w_{2}\right|} \Theta\left(w_{2}, w_{1}\right), \quad \forall w_{1}, w_{2} \in W .
$$

A symplectic structure on $W$ corresponds to the case $d=1$ and $\Theta$ non-degenerate.

The associated vector space of "cyclic words in $W$ ",

$$
C y c^{\bullet}(W):=\sum_{n \geq 0}\left(W^{\otimes n}\right)^{\mathbb{Z}_{n}},
$$

admits a $\mathcal{L} i e b_{d}^{\diamond}$-structure given by the following well-known formulae for the Lie bracket and cobracket,

$$
\begin{aligned}
& {\left[\left(w_{1} \otimes \ldots \otimes w_{n}\right)_{\mathbb{Z}_{n}},\left(w_{1}^{\prime} \otimes \ldots \otimes w_{m}^{\prime}\right)^{\mathbb{Z}_{n}}\right]:=} \\
& \sum_{i=1}^{n} \sum_{j=1}^{m} \pm \Theta\left(w_{i}, w_{j}^{\prime}\right)\left(w_{1} \otimes \ldots \otimes w_{i-1} \otimes w_{j+1}^{\prime} \otimes \ldots \otimes w_{m}^{\prime} \otimes w_{1}^{\prime} \otimes \ldots \otimes w_{j-1}^{\prime} \otimes w_{i+1} \otimes \ldots \otimes w_{n}\right)^{\mathbb{Z}_{n+m-2}} \\
& \triangle\left(w_{1} \otimes \ldots \otimes w_{n}\right)_{\mathbb{Z}_{n}}:=\sum_{i \neq j} \pm \Theta\left(w_{i}, w_{j}\right)\left(w_{i+1} \otimes \ldots \otimes w_{j-1}\right)^{\mathbb{Z}_{j-i-1}} \otimes\left(w_{j+1} \otimes \ldots \otimes w_{i-1}\right)^{\mathbb{Z}_{n-j+i-1}}
\end{aligned}
$$

where \pm stands for the standard Koszul sign. A very short (and pictorial) proof of this claim can be found in MW1. Note that the vector space $C y c^{\bullet}(W)$ is naturally weight-graded

$$
C y c^{\bullet}(W)=\bigoplus_{n \geq 0} C y c^{n}(W), \quad C y c^{n}(W):=\left(\otimes^{n} W\right)^{\mathbb{Z}_{n}}
$$

by the length of cyclic words, and both operations $\Delta$ and $[$,$] have weight-degree -2$ with respect to this weight-grading (which should not be confused with the homological grading).

2.5.1. A special case: Schedler's necklace Lie bialgebra. A special case of the above construction for $d=1$ gives us Schedler's necklace Lie bialgebra structure [S] associated with the quiver (11). Consider a set of $N$ formal letters

$$
\left\{x_{1}, \ldots, x_{n}\right\}
$$

and denote by $W_{N}$ their linear span over a field $\mathbb{K}$. We shall make $C y c(W)$ into a weight-degree -1 (not -2 as in the above example!) involutive Lie bialgebra using the following "doubling" trick.

Consider two copies $W_{N}^{(1)}, W_{N}^{(2)}$ of $W_{N}$ and equip their direct sum

$$
\hat{W}_{N}:=W_{N}^{(1)} \oplus W_{N}^{(2)}
$$

with the unique symplectic structure $\theta: \wedge^{2} \hat{W} \rightarrow \mathbb{K}$ making the basis $\left\{x_{\alpha}^{(1)}, x_{\beta}^{(2)}\right\}_{1 \leq \alpha, \beta \leq N}$ a Darboux one,

$$
\Theta\left(x_{\alpha}^{(1)}, x_{\beta}^{(2)}\right)=-\Theta\left(x_{\beta}^{(2)}, x_{\alpha}^{(1)}\right)=\delta_{\beta}^{\alpha}, \quad \Theta\left(x_{\alpha}^{(1)}, x_{\beta}^{(1)}\right)=0, \quad \Theta\left(x_{\alpha}^{(2)}, x_{\beta}^{(2)}\right)=0 .
$$

Then the above formulae for $[$,$] and \Delta$ make the space $C y c\left(\hat{W}_{N}\right)$ into an involutive Lie bialgebra. It is easy to see that the subspace

$$
\operatorname{Cyc}\left(\hat{W}_{N}\right)^{(12)} \subset \operatorname{Cyc}\left(\hat{W}_{N}\right)
$$


spanned by cyclic words of the form

$$
\left(x_{\alpha_{1}}^{(1)} \otimes x_{\alpha_{1}}^{(2)} \otimes x_{\alpha_{2}}^{(1)} \otimes x_{\alpha_{2}}^{(2)} \otimes \ldots \otimes x_{\alpha_{n}}^{(1)} \otimes x_{\alpha_{n}}^{(2)}\right)^{\mathbb{Z}_{2 n}}
$$

is closed with respect to the above Lie bracket and co-bracket. The canonical isomorphism

$$
\begin{array}{clc}
\operatorname{Cyc}\left(W_{N}\right) & \longrightarrow & \operatorname{Cyc}\left(\hat{W}_{N}\right)^{(12)} \\
\left(x_{\alpha_{1}} \otimes x_{\alpha_{2}} \otimes \ldots \otimes x_{\alpha_{n}}\right)^{\mathbb{Z}_{n}} & \longrightarrow & \left(x_{\alpha_{1}}^{(1)} \otimes x_{\alpha_{1}}^{(2)} \otimes x_{\alpha_{2}}^{(1)} \otimes x_{\alpha_{2}}^{(2)} \otimes \ldots \otimes x_{\alpha_{n}}^{(1)} \otimes x_{\alpha_{n}}^{(2)}\right)^{\mathbb{Z}_{2 n}}
\end{array}
$$

makes $C y c\left(W_{N}\right)$ into an involutive Lie bialgebra with the Lie bracket identical to the one introduced earlier by Schedler in $\underline{\mathrm{S}},[]=,[,]^{S}$, but with the Lie cobracket slightly different,

$$
\Delta\left(e_{\alpha_{1}} \otimes \ldots \otimes x_{\alpha_{n}}\right)^{\mathbb{Z}_{n}}=\Delta^{S}\left(x_{\alpha_{1}} \otimes \ldots \otimes x_{\alpha_{n}}\right)^{\mathbb{Z}_{n}}+\sum_{i=1}^{n} 1 \wedge\left(x_{\alpha_{1}} \otimes \ldots \otimes x_{\alpha_{i-1}} \otimes x_{\alpha_{i+1}} \otimes \ldots \otimes x_{\alpha_{n}}\right)^{\mathbb{Z}_{n-1}} .
$$

This purely combinatorial Lie bialgebra structure on $C y c\left(W_{N}\right)$ has a beautiful geometric interpretation - it is isomorphic AKKN1, AN, Ma to the Goldman-Turaev Lie bialgebra structure on the space of free loops in $\Sigma_{0, N+1}$, the two dimensional sphere with $n+1$ non-intersecting open disks removed.

\section{A prop of ribbon hypergraphs}

3.1. Ribbon hypergraphs. A ribbon hypergraph $\Gamma$ is, by definition, a triple $\left(E(\Gamma), \sigma_{1}, \sigma_{0}\right)$ consisting of a finite set $E(\Gamma)$ of edges and two arbitrary bijections ("permutations") $\sigma_{0}, \sigma_{1}: E(\Gamma) \rightarrow E(\Gamma)$. The orbits

$$
V(\Gamma):=E(\Gamma) / \sigma_{0}
$$

or, equivalently, the cycles of the permutation $\sigma_{0}$ are called the vertices of $\Gamma$ while the orbits

$$
H(\Gamma):=E(\Gamma) / \sigma_{1}
$$

of the permutation $\sigma_{1}$ are called hyperedges of $\Gamma$ (cf. [LZ). Let $p_{\circ}: E(\Gamma) \rightarrow V(\Gamma)$ and $p_{*}: E(\Gamma) \rightarrow H(\Gamma)$ be canonical projections. For any vertex $v \in V(\Gamma)$ and any hyperedge $h \in H(\Gamma)$ the associated sets of edges

$$
p_{\circ}^{-1}(v)=\left\{e_{i_{1}}, \ldots, e_{i_{k}}\right\}, \quad p_{*}^{-1}(h)=\left\{e_{j_{1}}, \ldots, e_{j_{l}}\right\}, \quad k, l \in \mathbb{N}
$$

come equipped with induced cyclic orderings and hence can be represented pictorially as planar corollas,

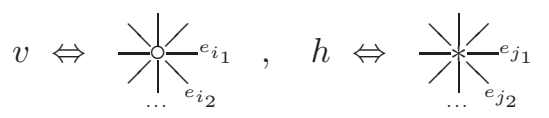

Each edge $e \in E(\Gamma)$ belongs to precisely one vertex, $e \in p_{0}^{-1}(v)$ for some $v \in V(\Gamma)$, and precisely one hyperedge, $e \in p_{*}^{-1}(h)$ for some $h \in H(\Gamma)$. Hence we can glue the vertex $v$ to the corresponding hyperedge $h$ along the the common edge(s) to get a pictorial ( $C W$ complex like) representation of a ribbon hypergraph $\Gamma$ as an ordinary ribbon graph whose vertices are bicoloured, e.g.

$$
\Gamma_{1}=\overbrace{0}^{*}, \quad \Gamma_{2}=\circlearrowright \quad, \quad \Gamma_{3}=* \longrightarrow
$$

The vertices of $\Gamma$ get represented pictorially as white vertices while hyperedges as asterisk vertices; sometimes we call a hyperedge an asterisk vertex when commenting some pictures. Note that each edge $e \in E(\Gamma)$ connects precisely one white vertex to precisely one asterisk vertex; such bicoloured ribbon graphs are called hypermaps in [LZ.

Note that the set of edges $E(\Gamma)$ admits two decompositions into disjoint subsets,

$$
E(\Gamma)=\coprod_{v \in V(\Gamma)} p_{\circ}^{-1}(v), \quad E(\Gamma)=\coprod_{h \in H(\Gamma)} p_{*}^{-1}(h)
$$

with each subset having an induced cyclic ordering. 
The orbits of the permutation $\sigma_{\infty}:=\sigma_{0}^{-1} \circ \sigma_{1}$ are called boundaries of the ribbon hypergraph $\Gamma$; the set of boundaries is denoted by $B(\Gamma)$. For example, in the case of the above ribbon hypergraphs we have $\# V\left(\Gamma_{1}\right)=3, \# H\left(\Gamma_{1}\right)=1, \# B\left(\Gamma_{1}\right)=1, \# E\left(\Gamma_{1}\right)=3, \# V\left(\Gamma_{2}\right)=1, \# H\left(\Gamma_{2}\right)=1, \# B\left(\Gamma_{2}\right)=3, \# E\left(\Gamma_{2}\right)=3$ and $\# V\left(\Gamma_{3}\right)=1, \# H\left(\Gamma_{3}\right)=2, \# B\left(\Gamma_{3}\right)=2, \# E\left(\Gamma_{3}\right)=3$. For a vertex $v \in V(\Gamma)$ (resp, a hyperedge $h \in H(\Gamma)$ ) we denote its valency by $|v|:=\# p_{\circ}^{-1}(v)$ (resp., $|h|:=\# p_{*}^{-1}(h)$ ).

A ribbon hypergraph with each hyperedge having valency 2 is called a ribbon graph. Ribbon graphs are depicted pictorially with asterisk vertices omitted as they contain no extra information,

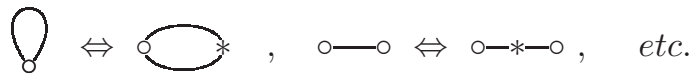

3.2. On geometric interpretation of ribbon hypergraphs. Every connected ribbon graph $\Gamma$ can be interpreted geometrically as a topological 2-dimensional surface with $\# B(\Gamma)$ boundary circles and $\# V(\Gamma)$ punctures which is obtained from its $C W$-complex realization by thickening its every vertex into a closed disk punctured in the center and then thickening its every edge $e \in E(\Gamma)$ into a 2-dimensional strip. For example (cf. MW1),

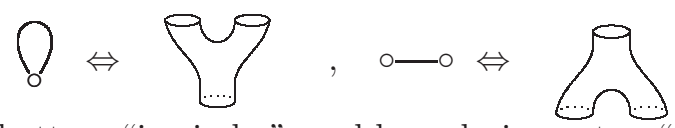

with punctures represented as bottom "in-circles", and boundaries as top "out-circles".

A ribbon hypergraph $\Gamma$ is often used [LZ] to encode combinatorially a Belyi map, that is, a ramified covering $f: X \rightarrow \mathbb{C P}^{1}$ of the sphere whose ramification locus is contained in the set $\{0,1, \infty\}$. A famous Belyi theorem says that every smooth projective algebraic curve $X$ defined over the algebraic closure $\overline{\mathbb{Q}}$ (in $\mathbb{C}$ ) of rational numbers can be realized as such a ramified covering of $\mathbb{C P}^{1}$. Moreover, the universal Galois group Gal( $\overline{\mathbb{Q}}: \mathbb{Q})$ acts faithfully on (equivalence classes of) Belyi maps. Given a Belyi map $f: X \rightarrow \mathbb{C P}^{1}$, the associated ribbon hypergraph (dessin d'enfants) $\Gamma$ is embedded into the Riemann surface $X$ as the pre-image

$$
f^{-1}(\bullet-\circ)
$$

of the unit interval $[0,1] \subset \mathbb{C P}^{1}$ with the point 0 presented as the white vertex vertex and the point 1 as the asterisk. For example, the hypergraph

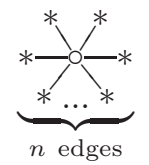

corresponds to the Belyi map $f: \mathbb{C P}^{1} \rightarrow \mathbb{C P}^{1}$ given by $f(z)=z^{n}$, while the hypergraph

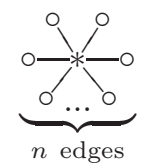

corresponds to the Belyi map $f(z)=(1-z)^{n}$. However, ribbon hypergraphs used in this paper are $\mathbb{Z}$ graded and oriented (see the next subsection) and it is not clear how this extra structure may fit this particular geometric interpretation of hypergraphs. Oriented ribbon graphs have a useful interpretation as combinatorial objects parameterizing cells of a certain cell decomposition of the moduli spaces $\mathcal{M}_{g, n}$ of algebraic curves of genus $g$ with $n$ punctures; they also play an important role in the deformation theory of the Goldman-Turaev Lie bialgebra. We show in this paper that oriented ribbon hypergraphs have much to do with strongly homotopy involutive Lie bialgebras and hence might be useful in the string topology.

3.3. Orientation and $\mathbb{Z}$ grading. Let $d$ be an arbitrary integer. To a ribbon hypergraph $\Gamma$ we assign its homological degree

$$
|\Gamma|=(d+1) \# H(\Gamma)-d \# E(\Gamma)
$$

i.e. each hyperedge has degree $d+1$ and every edge has degree $-d$.

An orientation on a ribbon hypergraph $\Gamma$ is, by definition, a

- choice of the total ordering (up to an even permutation) on the set of hyperedges $H(\Gamma)$ for $d$ even, 
- choice of the compatible (with the underlying cyclic ordering) total ordering — up to an even permutation — on each subset of edges $p_{*}^{-1}(h) \subset E(\Gamma), \forall h \in H(\Gamma)$, for $d$ odd.

As $E(\Gamma)=\sqcup_{h \in H(\Gamma)} p_{*}^{-1}(h)$ and each set $p_{*}^{-1}(h)$ is cyclically ordered, a choice of the total ordering in $E(\Gamma)$ for $d$ odd is equivalent to a choice of a total ordering of $H_{\text {odd }}(\Gamma):=\{h \in H(\Gamma)|| h \mid \in 2 \mathbb{Z}+1\}$ (up to an even permutation), and a choice of a total ordering of each set $p_{*}^{-1}(h), h \in H_{\text {even }}(\Gamma):=\{h \in H(\Gamma)|| h \mid \in 2 \mathbb{Z}\}$ which is compatible with the given cyclic ordering (again up to an even permutation).

Note that every ribbon hypergraph has precisely two possible orientations. If $\Gamma$ is an oriented ribbon hypergraph, then the same hypergraph equipped with an opposite orientation is denoted by $\Gamma^{o p p}$.

Also note that if $\Gamma$ has all hyperedges bivalent, then the above definition agrees with the notion of orientation in the prop of ribbon graphs $\mathcal{R} \mathcal{G} r a_{d+1}$ introduced in [MW].

3.4. Boundaries and corners of a hypergraph. Let us represent pictorially a ribbon hypergraph $\Gamma$ with vertices and hyperedges blown up into dashed and, respectively, double solid circles, for example

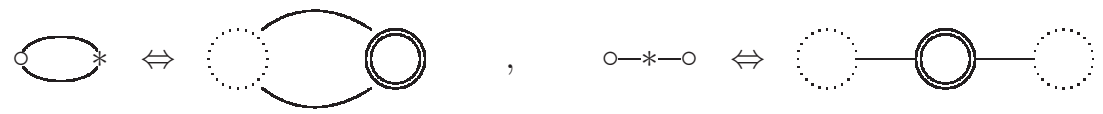

Edges attached to dashed (resp. double solid) circles divide the latters into the disjoint union of chords which can be called vertex (resp. hyperedge) corners; thus with any vertex $v \in V(\Gamma)$ we associate a cyclically ordered set $C(v)$ of its corners (which is, of course, isomorphic to its set $p_{\circ}^{-1}(v)$ of attached edges but has a different geometric incarnation). The motivation for this terminology is that any boundary of a hypergraph can be understood as a polytope glued from edges at that corners. For example, the unique boundary $b$ of the right graph just above is given the following polytope

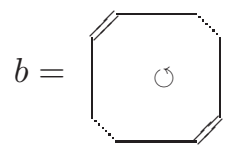

where small dashed (resp. double solid) intervals stand for the vertex (resp. hyperedge) corners. Thus with any boundary $b$ of a hypergraph one can associate a cyclically ordered set $C(b)$ of its vertex corners. We shall use these cyclically ordered sets $C(v)$ and $C(b)$ in the definition of the prop composition of hypergraphs below, and in the construction of canonical representations of that prop in spaces of cyclic words.

3.5. Prop of ribbon hypergraphs. Let $\mathcal{R} \mathcal{H}_{m, n}^{k, l}$ be the set of (isomorphism classes of) oriented ribbon hypergraphs $\Gamma$ with $n$ vertices labelled by elements of $[n], k$ edges, $l$ unlabelled hyperedges and $m$ boundaries labelled by elements of $[m]$. Consider a collection of quotient $\mathbb{S}$-bimodules,

$$
\mathcal{R} \mathcal{H r a}_{d}:=\left\{\mathcal{R} \mathcal{H} r a_{d}(m, n):=\bigoplus_{k, l \geq 1} \frac{\mathbb{K}\left\langle\mathcal{R H}_{m, n}^{k, l}\right\rangle}{\left\{\Gamma=-\Gamma^{o p p}, \Gamma \in \mathcal{H}_{m, n}^{k, l}\right\}}[d k-(d+1) l]\right\}_{m, n \geq 1}
$$

Thus elements of $\mathcal{R H}$ ra are isomorphisms classes of $\mathbb{Z}$-graded oriented ribbon hypergraphs $\Gamma$ whose vertices and boundaries are enumerated. Ribbon hypergraphs admitting automorphisms which reverse their orientations are equal to zero in $\mathcal{R} \mathcal{H} r a_{d}$. The $\mathbb{S}$-module $\mathcal{R} \mathcal{H} r a_{d}$ contains a submodule $\mathcal{R} \mathcal{G} r a_{d}$ generated by hypergraphs with all hyperedges bivalent. This submodule has a prop structure [MW1] which can be easily extended to $\mathcal{R} \mathcal{H} r a_{d}$. Indeed, the horizontal composition

$$
\begin{array}{ccc}
\circ: \mathcal{R H r a}_{d}\left(m_{1}, n_{1}\right) \otimes_{\mathbb{K}} \mathcal{R H r a}\left(m_{2}, n_{2}\right) & \longrightarrow & \mathcal{R H r a} a_{d}\left(m_{1}+m_{2}, n_{1}+n_{2}\right) \\
\Gamma_{2} \otimes \Gamma_{1} & \longrightarrow & \Gamma_{2} \sqcup \Gamma_{1}
\end{array}
$$

is defined as the disjoint union of ribbon hypergraphs, and the vertical composition,

$$
\begin{aligned}
& \circ: \quad \mathcal{R} \mathcal{H} r a_{d}(p, m) \otimes_{\mathbb{K}} \mathcal{R} \mathcal{H} r a_{d}(m, n) \longrightarrow \mathcal{R} \mathcal{H} r a_{d}(p, n) \\
& \Gamma_{2} \otimes \Gamma_{1} \quad \longrightarrow \quad \Gamma_{2} \circ \Gamma_{1}
\end{aligned}
$$


is defined by gluing, for every $i \in[m]$, the $i$-th oriented boundary $b$ of $\Gamma_{1}$,

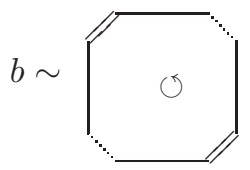

with the $i$-th vertex $v$ of $\Gamma_{2}$,

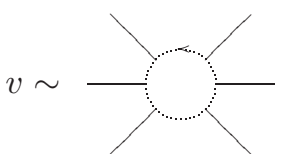

by erasing the vertex $v$ from $\Gamma_{2}$ and taking the sum over all possible ways of attaching "hanging in the air" (half)edges from the set $p_{\circ}^{-1}(v)$ to the set of dashed corners from $C(b)$ while respecting the cyclic structures of both sets; put another way we take a sum over all morphisms $p_{\circ}^{-1}(v) \rightarrow C(b)$ of cyclically ordered sets. Every ribbon graph in this linear combination comes equipped naturally with an induced orientation, and belongs to $\mathcal{R} \mathcal{H} r a_{d}(p, n)$. The graph o consisting of a single white vertex acts as the unit in $\mathcal{R} \mathcal{H} r a_{d}$. The subspace of $\mathcal{R} \mathcal{H} r a_{d}$ spanned by connected ribbon graphs forms a properad which we denote by the same symbol $\mathcal{R H} r a_{d}$.

3.5.1. Theorem. Let $W$ be an arbitrary graded vector space and $C y c(W)=\oplus_{n \geq 0}\left(W^{\otimes n}\right)^{\mathbb{Z}_{n}}$ the associated space of cyclic words. Then any collection

$$
\Theta_{n}:(W[d])_{\mathbb{Z}_{n}}^{\otimes n} \longrightarrow \mathbb{K}[1+d], \quad n \geq 1,
$$

of cyclically (skew)invariant maps gives canonically rise to a representation

$$
\rho_{\Theta}: \mathcal{R} \mathcal{H} r a_{d} \longrightarrow \mathcal{E} n d_{C y c(W)} .
$$

of the prop of hypergraphs in $C y c(W)$.

Proof. If only $\Theta_{2}$ is non-zero, the associated representation

$$
\rho_{\Theta_{2}}: \mathcal{R} \mathcal{G} r a_{d} \longrightarrow \mathcal{E} n d_{C y c(W)}
$$

was constructed in Theorem 4.2.2 of [MW1. The general case is a straightforward hypergraph extension of that construction. Let us sketch this extension for $d$ even (the case $d$ odd is completely analogous). Consider a hypergraph $\Gamma \in \mathcal{R} \mathcal{H} r a_{d}(m, n)$ with $n$ vertices $\left(v_{1}, \ldots, v_{i}, \ldots, v_{n}\right)$ and $m$ boundaries $\left(b_{1}, \ldots, b_{j}, \ldots, b_{m}\right)$. Using $\Theta_{\bullet}$ we will construct a linear map

$$
\begin{array}{cccc}
\rho_{\Theta}^{\Gamma}: & \otimes^{n} C y c(W) & \longrightarrow & \otimes^{m} C y c(W) \\
& \mathcal{W}_{1} \otimes \ldots \otimes \mathcal{W}_{n} & \longrightarrow & \rho_{\Theta}^{\Gamma}\left(\mathcal{W}_{1}, \ldots, \mathcal{W}_{n}\right)
\end{array}
$$

where

$$
\left\{\mathcal{W}_{i}:=\left(w_{i_{1}} \otimes \ldots \otimes w_{p_{i}}\right)_{\mathbb{Z}_{p_{i}}}\right\}_{1 \leq i \leq n, \quad p_{i} \in \mathbb{N}}
$$

is an arbitrary collection of $n$ cyclic words from $C y c(W)$. If $\# p_{\circ}^{-1}\left(v_{i}\right)>p_{i}$ for at least one $i \in[n]$, i.e. if number of edges attached to $v_{i}$ greater than the length of the word $\mathcal{W}_{i}$, we set $\rho_{\Theta}^{\Gamma}\left(\mathcal{W}_{1}, \ldots, \mathcal{W}_{n}\right)=0$. Otherwise it makes sense to consider a state $s$ which is by definition a collection of fixed injective morphisms of cyclically ordered sets

$$
s_{i}: p_{\circ}^{-1}\left(v_{i}\right) \longrightarrow\left\{w_{i_{1}}, \ldots, w_{p_{i}}\right\}, \quad \forall i \in[n],
$$

that is, an assignment of some letter $w_{i}$ from the word $\mathcal{W}_{i}$ to each edge $e_{i} \in p_{\circ}^{-1}\left(v_{i}\right)$ of each vertex $v_{i}$ in a way which respects cyclic orderings of both sets. Note that for each state the complement, $\left(w_{i_{1}}, \ldots, w_{i_{l_{p}}}\right) \backslash \operatorname{Im} s_{i}$, splits into a disjoint (cyclically ordered) union of totally ordered subsets, $\coprod_{c \in C(v)} I_{c}$, parameterized by the set of corners of the vertex $v$. Note also that to each boundary $b_{j} \in B(\Gamma)$ we can associate a cyclic word

$$
\mathcal{W}_{j}^{\prime}:=\left(\bigotimes_{c \in C\left(b_{j}\right)} I_{c}\right)_{\mathbb{Z}_{\sum_{c \in C\left(b_{j}\right)} \# I_{c}}}
$$


where the tensor product is taken along the given cyclic ordering in the set $C\left(b_{j}\right)$,

Recall that the set of hyperedges $H(\Gamma)$ is defined as the set of orbits $E(\Gamma) / \sigma_{1}$ of the permutation $\sigma_{1}$. To any hyperedge $h \in H(\Gamma)$ of valency $|h| \in \mathbb{N}$ there corresponds therefore a cyclically ordered set of $|h|$ edges $p_{*}^{-1}(h) \subset E(\Gamma)$. Let us choose for a moment a compatible total order on this set, i.e. write it as

$$
p_{*}^{-1}(h)=\left\{e_{1}^{h}, e_{2}^{h}=\sigma_{1}\left(e_{1}^{h}\right), \ldots, \ldots e_{|h|}^{h}=\sigma_{1}^{|h|-1}\left(e_{1}^{h}\right)\right\}
$$

for some chosen edge $e_{1}^{h} \in p_{*}^{-1}(h)$. As the set of edges decomposes into the disjoint union

$$
E(\Gamma)=\coprod_{h \in H(\Gamma)} p_{*}^{-1}(h),
$$

we can use the given maps $\Theta_{k}:\left(\otimes^{k} W\right)_{\mathbb{Z}_{k}} \rightarrow \mathbb{K}[1+d-d k]$ to define the weight of any given state $s$ on $\Gamma$ as the following number,

$$
\lambda_{s}:=\prod_{h \in H(\Gamma)} \Theta_{|h|}\left(s\left(e_{1}^{h}\right), \ldots s\left(e_{|h|}^{h}\right)\right) .
$$

Thus to each state $s=\left\{s_{i}\right\}_{i \in[n]}$ we associate an element

$$
\rho_{\Theta}^{s}\left(\mathcal{W}_{1}, \ldots, \mathcal{W}_{n}\right):=(-1)^{\sigma} \lambda_{s} \mathcal{W}_{b_{1}}^{\prime} \otimes \ldots \otimes \mathcal{W}_{b_{m}}^{\prime} \in \otimes^{m} C y c(W)
$$

where $(-1)^{\sigma}$ is the standard Koszul sign of the regrouping permutation,

$$
\sigma: \mathcal{W}_{1} \otimes \ldots \otimes \mathcal{W}_{n} \longrightarrow \prod_{h \in(\Gamma)}\left(s\left(e_{1}^{h}\right), \ldots s\left(e_{|h|}^{h}\right)\right) \otimes \mathcal{W}_{b_{1}}^{\prime} \otimes \ldots \otimes \mathcal{W}_{b_{m}}^{\prime} .
$$

Note that $\rho_{\Theta}^{s}\left(\mathcal{W}_{1}, \ldots, \mathcal{W}_{n}\right)$ does not depend on the choices of compatible total orderings in the sets $p_{*}^{-1}(h)$ made above.

Finally we define a linear map,

$$
\begin{array}{ccc}
\rho_{\Theta}: \quad \mathcal{R G r a}_{d}(m, n) & \longrightarrow & \operatorname{Hom}\left(\otimes^{n} C y c(W), \otimes^{m} C y c(W)\right) \\
\Gamma & \longrightarrow & \rho_{\Theta}^{\Gamma}
\end{array}
$$

by setting the value of $\rho_{\Theta}^{\Gamma}$. on cyclic words (13) to be equal to

$$
\rho_{\Theta}^{\Gamma}\left(\mathcal{W}_{1}, \ldots, \mathcal{W}_{n}\right):=\left\{\begin{array}{cl}
0 & \text { if } \# p_{\circ}^{-1}\left(v_{i}\right)>p_{i} \text { for some } i \in[n] \\
\sum_{\substack{\text { all possible } \\
\text { states } s}} \rho_{\Theta \bullet}^{s}\left(\mathcal{W}_{1}, \ldots, \mathcal{W}_{n}\right) & \text { otherwise }
\end{array}\right.
$$

It is now straightforward to check that the map $\rho_{\Theta}$. respects prop compositions in $\mathcal{R} \mathcal{H} r a_{d}$ and $\mathcal{E} n d_{C y c(W)}$ because the prop structure in former has been just read off from the compositions of operators $\rho_{\Theta}^{\Gamma}$. in the latter.

\section{Strongly homotopy involutive Lie bialgebras and ribbon hypergraphs}

4.1. Reminder from MW1. There is a morphism of props,

$$
\rho: \mathcal{L} i e b_{d}^{\diamond} \longrightarrow \mathcal{R H} \mathrm{Hra}_{d}
$$

given on generators as follows,

$$
\rho(Y)=\underbrace{*}, \rho(\boldsymbol{d})=0-*-\circ
$$

The main new result of this note is an observation that this maps lifts to a morphism of dg props $\mathcal{H}$ olieb ${ }_{d}^{\diamond} \rightarrow$ $\mathcal{R} \mathcal{H} r a_{d}$ which is non-trivial on all generators (see below). 
4.2. Proposition. There is a morphism of $d g$ props,

$$
\rho: \mathcal{H} \text { olieb }_{d} \longrightarrow \mathcal{R} \mathcal{H} r a_{d}
$$

given on generators as follows,

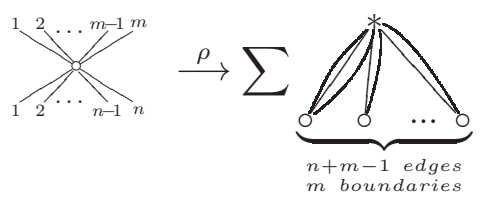

where the sum on the right hand side is over all possible ways of attaching $n+m-1$ edges beginning at the asterisk vertex to $n$ white vertices (whose numerical labels are (skew)symmetrized) in such a way that every white vertex is hit and the total number of boundaries of the resulting hypergraph equals precisely $m$ (and their numerical labels are also (skew)symmetrized).

Proof. As the prop $\mathcal{R} \mathcal{H} r a_{d}$ has vanishing differential, the Propsoition holds true if and only of

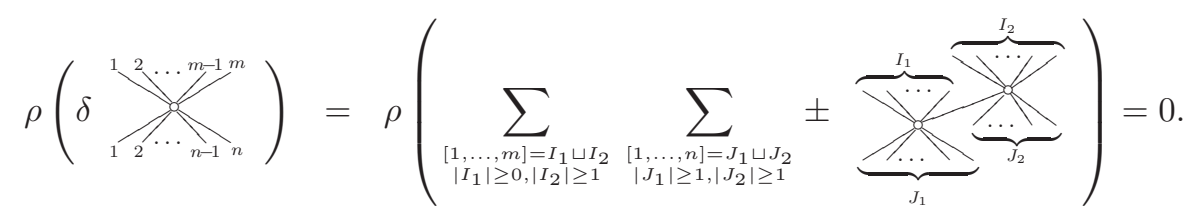

This is almsot obvious as the r.h.s. is given by the sum

$$
\sum_{\substack{[1, \ldots, m]=I_{1} \sqcup I_{2} \\\left|I_{1}\right| \geq 0,\left|I_{2}\right| \geq 1}} \sum_{\substack{[1, \ldots, n]=J_{1} \sqcup J_{2} \\\left|J_{1}\right| \geq 1,\left|J_{2}\right| \geq 1}} \sum \pm
$$

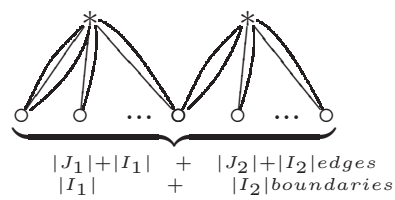

which vanishes in $\mathcal{R H} \mathrm{Ha}_{d}$ for symmetry reasons (it is most easy to check this claim in the case $d$ is even when the asterisk vertices are odd, and the symbol \pm becomes + ).

There is a canonical morphism of props $\mathcal{H}$ lieb $_{d} \rightarrow \mathcal{H}$ olieb ${ }_{d}^{\diamond}$. The above morphism factors through the composition Holieb $_{d} \rightarrow \mathcal{H}$ olieb $_{d}^{\diamond} \stackrel{\rho^{\diamond}}{\longrightarrow} \mathcal{R} \mathcal{H} r a_{d}$.

4.3. Theorem. There is a morphism of $d g$ props,

$$
\rho^{\diamond}: \mathcal{H} \text { olieb }_{d}^{\diamond} \longrightarrow \mathcal{R H} \mathcal{H r a}_{d}
$$

given on generators as follows,

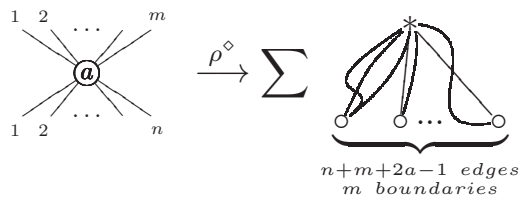

where the sum on the right hand side is over all possible ways to attach $n+m+2 a-1$ edges beginning at the asterisk vertex to $n$ white vertices (whose numerical labels are (skew)symmetrized) in such a way that every white vertex is hit and the total number of boundaries of the resulting hypergraph equals precisely $m$ (and their numerical labels are (skew)symmetrized).

The orientations of the hypergraphs shown in the above formula are determined uniquely by a simple $\mathcal{H o B} \mathcal{V}_{d}^{c o m}$ operator which is constructed in the proof.

Proof. The composition of the above map $\rho^{\diamond}$ with the canonical representation $\rho_{\Theta}$. from Proposition 3.5.1 implies that for any collection of cyclically (skew)symmetric maps 3 on a graded vector space $W$ the associated vector space $C y c(W)$ is canonically a $\mathcal{H}$ olieb ${ }_{d}^{\diamond}$ algebra. In fact one can read the Theorem from this conclusion provided the latter is independent of choices of $W$ and the higher products $\Theta_{\bullet}$. 
Assume $d$ even. To make the construction of the representation $\rho_{\Theta} \circ \rho^{\diamond}$ as simple and transparent as possible we shall employ, following Serguei Barannikov [Ba2, the invariant theory and identify the space $C y c(W)$ with the space of $G L\left(\mathbb{K}^{N}\right)$-invariants,

$$
C y c(W)=\lim _{N \rightarrow \infty} \oplus_{n \geq 0}\left(\otimes^{n}\left(W \otimes \operatorname{End}\left(\mathbb{K}^{n}\right)\right)^{G L\left(\mathbb{K}^{N}\right)},\right.
$$

that is, we interpret a cyclic word, $\mathcal{W}=\left(w_{a_{1}} \otimes \ldots \otimes w_{a_{n}}\right)^{\mathbb{Z}_{n}},\left\{w_{i}\right\}_{i \in I}$ a basis in $W$, with the trace of the product of $N \times N$ matrices,

$$
\mathcal{W}=\operatorname{tr}\left(A_{a_{1}} A_{a_{2}} \cdots A_{a_{n}}\right)=\sum_{\alpha_{\bullet}} A_{a_{1} \alpha_{1}}^{\alpha_{0}} A_{a_{2} \alpha_{2}}^{\alpha_{1}} \cdots A_{a_{n} \alpha_{0}}^{\alpha_{n}}, \quad A_{i_{a}}:=\left(A_{i_{a} \beta}^{\alpha}\right) \in \operatorname{End}\left(\mathbb{K}^{N}\right), \quad a \in[n], \alpha, \beta \in[N],
$$

for sufficiently large $N \in \mathbb{N}$. The graded cyclically symmetric maps

$$
\begin{array}{cccc}
\Theta_{n}: & \otimes^{n} W & \longrightarrow & \mathbb{K}[1+d-n d] \\
& w_{a_{1}} \otimes \ldots \otimes w_{a_{n}} & \longrightarrow & \Theta_{a_{1} \ldots a_{n}}
\end{array}
$$

define a degree 1 operator

$$
\Delta=\sum_{n \geq 1} \hbar^{n-1} \Theta_{a_{1} \ldots a_{n}} \frac{\partial^{n}}{\partial A_{a_{1} \alpha_{1}}^{\alpha_{0}} \partial A_{a_{2} \alpha_{2}}^{\alpha_{1}} \cdots \partial A_{a_{n} \alpha_{0}}^{\alpha_{n}}}
$$

on $\odot \bullet(C y c(W)[-d])[[\hbar]]$ whose square is obviously zero; here the formal parameter $\hbar$ has degree $2 d$. The latter defines a $\mathcal{H o B V}_{d}^{\text {com }}$-structure in $\left.\odot \bullet(C y c(W)[-d])[\hbar]\right]$ and, as explained in 2.4 an associated $\mathcal{H} o l i e b_{d}^{\diamond}\{d\}$ algebra structure in $C y c(W)[-d]$ which in turn defines a $\mathcal{H}$ olieb ${ }_{d}^{\diamond}$-structure in $C y c(W)$.

If $d$ is odd, one can again use a trick from $\mathrm{Ba2}$, which replaces the ordinary trace of the standard matrix superalgebra with the odd trace of the Bernstein-Leites matrix sub-superalgebra. In fact the construction in $\mathrm{Ba2}$ explains the construction of the $\mathcal{H o B V}_{d}^{c o m}$ operator in the case when only $\Theta_{2}$ is non-zero, and its extension to the general case (3) is completely analogous to the $d$ even case discussed above.

4.4. Corollary. Let $W$ be a graded vector space equipped with a collection of linear maps

$$
\Theta_{p}: \otimes^{p}(W[d])_{\mathbb{Z}_{p}} \longrightarrow \mathbb{K}[1+d], \quad p \geq 2,
$$

Each map $\Theta_{p}$ makes the (reduced) graded vector space of cyclic words in $W$,

$$
C y c(W)=\oplus_{n \geq 0}\left(\otimes^{n} W\right)_{\mathbb{Z}_{n}} \text { or } \overline{C y c}(W)=\oplus_{n \geq 1}\left(\otimes^{n} W\right)_{\mathbb{Z}_{n}},
$$

into a $\mathcal{H}_{\text {olieb }}^{\diamond}$-algebra with only those $\mathcal{H}$ olieb $b_{d}^{\diamond}$-operations

$$
\mu(m, n, a) \simeq \underbrace{1 \ldots m_{n-1}^{m}}_{12 \ldots-1 n}: \otimes^{n} C y c(W) \longrightarrow \otimes^{m} C y c(W), \quad m \geq 1, n \geq 1, m+n+2 a \geq 3,
$$

non-trivial which satisfy the condition $m+n+2 a=p+1$. Moreover, this $\mathcal{H}_{\text {olieb }}{ }_{d}^{\diamond}$-algebra structure admits an independent rescaling automorphism

$$
\mu(m, n, a) \rightarrow \lambda_{m+n+2 a} \cdot \mu(n, m, a), \quad \lambda_{m+n+2 a} \in \mathbb{K}^{*},
$$

for each fixes sum $m+n+2 a$ of the integer parameters.

In the case $p=2$ this construction reproduces the classical construction of $\mathcal{L}_{i e} b_{d}^{\diamond}$-algebra structure on $C y c(W)$ given in 2.5 .

4.5. Rescaling freedom. Note that each map $\Theta_{k}$ from the family (3) can be independently rescaled, $\Theta_{k} \rightarrow \lambda_{k} \Theta_{k}, \forall \lambda_{n} \in \mathbb{K}$, so that the morphism $\rho^{\diamond}$ in (14) can also be rescaled by infinitely many independent parameters - just rescale in that formula each hyperedge $h$ of valency $k$ by

$$
h=\underset{\nearrow}{\nearrow} \backslash \longrightarrow \lambda_{k} \cdot \underset{\nearrow}{\nearrow} \backslash \quad, \forall \lambda_{k} \in \mathbb{K}
$$

and get a new morphism $\rho_{\lambda}^{\diamond}$ from $\mathcal{H}$ olieb ${ }_{d}^{\diamond}$ to $\mathcal{R} \mathcal{H} r a_{d}$. Such a phenomenon occurs in the string topology - see Theorem 6.2 and Corollary 6.3 in [CS - and its main technical origin in our case is that the prop $\mathcal{R} \mathcal{H} r a_{d}$ has vanishing differential. 


\section{An algebraic application: a new family of combinatorial $\mathcal{H}$ olie $b^{\diamond}$-algebras}

Given any collection of formal letters $e_{1}, \ldots, e_{N}$, i.e. given any natural number $N \geq 1$, there is an associated involutive Lie bialgebra structure on the vector space $C y c\left(W_{N}\right)$ of cyclic words,

$$
W_{N}:=\operatorname{span}_{\mathbb{K}}\left\langle e_{1}, e_{2}, \ldots, e_{N}\right\rangle
$$

which belongs to the family of combinatorial $\mathcal{L} i e b^{\diamond}$-algebras constructed by Schedler in $[\underline{\mathrm{S}}$ out of any quiver. This particular $\mathcal{L} i e b^{\diamond}$-algebra has an important geometric meaning (discussed in $\S 1$ ) and corresponds to the quiver (1).

In this section we use Theorem 4.3 and Corollary 6.3.1 in the case $d=1$ to extend that particular Schedler's construction to a highly non-trivial (i.e. with all higher homotopy operations non-zero) $\mathcal{H}$ olieb $b^{\diamond}$-algebra structure on the vector space $C y c\left(\widehat{W}_{N}\right)$ of cyclic words generated by $\mathbb{Z}$-graded formal letters,

$$
\widehat{W}_{N}:=\operatorname{span}_{\mathbb{K}}\left\langle e_{1}[-p], \ldots, e_{N}[-p]\right\rangle_{p \in \mathbb{N}}=\bigoplus_{p \geq 0} W_{N}[-p]
$$

where $e_{\alpha}[-p], \alpha \in[N]$, stands for the copy of the formal letter $e_{\alpha}$ to which we assign the homological degree $p$. Note that $\widehat{W}_{N}$ has no natural higher products (3) so we can not apply Corollary 6.3.1 immediately. The idea of our construction is to inject first

$$
u: \widehat{W}_{N} \longrightarrow \widehat{\mathbf{W}}_{N}
$$

$\widehat{W}_{N}$ into a larger space $\widehat{\mathbf{W}}_{N}$ which does have a natural family of cyclically (skew)symmetric higher products

$$
\left\{\Theta_{k+2}:\left(\otimes^{k+2}\left(\widehat{\mathbf{W}}_{N}[1]\right)\right)_{\mathbb{Z}_{k+2}} \longrightarrow \mathbb{K}[2]\right\}_{k \geq 0}
$$

so that the associated vector space of cyclic words $C y c\left(\widehat{\mathbf{W}}_{N}\right)$ comes equipped with a canonical Holieb ${ }^{\diamond}$ algebra structure by Corollary 6.3.1. The second step will be to check that the image of $u$ is closed with respect to all strongly homotopy involutive Lie bialgebra operations. To realize this programme, consider, for any integer $p \geq 0$, a set of $p+2$ copies of the vector space $W_{N}$

$$
W_{N}^{0_{p}}:=W_{N}[-p], W_{N}^{1_{p}}:=W_{N}, \ldots, W_{N}^{p+1_{p}}:=W_{N},
$$

one of them (say, labelled by zero) assigned a shifted homological degree, and define

$$
\widehat{\mathbf{W}}_{N}:=\bigoplus_{p \geq 0} W_{N, p}, \quad W_{N, p}:=W_{N}^{0_{p}} \oplus W_{N}^{1_{p}} \oplus \ldots \oplus \ldots W_{N}^{p+1_{p}}
$$

The vector space $\widehat{\mathbf{W}}_{N}$ is countably dimensional, and is equipped by construction with a distinguished basis

$$
\left\{e_{\alpha}^{l_{p}}\right\}_{p \geq 0,0 \leq l \leq p+1,1 \leq \alpha \leq N}
$$

where $\left\{e_{\alpha}^{l_{p}}\right\}_{\alpha \in[N]}$ stands for the standard basis of the copy $W_{N}^{l_{p}}$. Note that for any $\alpha \in N$ the basis vector $e_{\alpha}^{l_{p}}$ has homological degree 0 if $l \geq 1$, and $-p$ of $l=0$. Note also that summands $W_{N}^{l_{p^{\prime}}}$ and $W_{N}^{l_{p^{\prime \prime}}}$ in $\widehat{\mathbf{W}}_{N}$ are viewed as different copies of $W_{N}$ for $p^{\prime} \neq p^{\prime \prime}$ (as they belong to different vector spaces $W_{N, p^{\prime}}$ and $W_{N, p^{\prime \prime}}$ ).

Let us introduce next an infinite family of cyclically (skew)symmetric higher products (the case $d=1$ in the notation (3))

$$
\Theta_{k+2}: \otimes^{k+2} \hat{\mathbf{W}}_{N} \longrightarrow \mathbb{K}[-k], \quad \forall k \geq 0,
$$

by setting

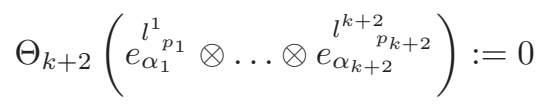

unless

(i) $\alpha_{1}=\alpha_{2}=\ldots=\alpha_{k+1}$;

(ii) $p_{1}=p_{2}=\ldots=p_{k+2}=k$; 
(iii) $\left(l^{1}, \ldots, l^{k+2}\right)=(j, j-1, \ldots, 2,1,0, k+1, k, \ldots, j+1)$ for some $j \in\{0,1,2, \ldots, k+1\}$, i.e. we have an isomorphism of cyclically ordered sets
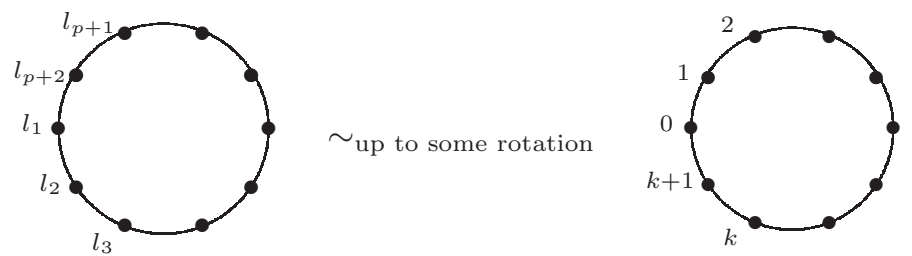

If all the above conditions are satisfied, we set

$$
\Theta_{k+2}\left(e_{\alpha}^{j_{k}} \otimes e_{\alpha}^{j-1_{k}} \otimes \ldots e_{\alpha}^{1_{k}} \otimes e_{\alpha}^{0_{p}} \otimes e_{\alpha}^{k+1_{k}} \otimes e_{\alpha}^{k_{k}} \otimes \ldots \otimes e_{\alpha}^{j+1_{k}}\right):=(-1)^{j(k+1)}
$$

By Corollary 6.3.1, the graded vector space $C y c^{\bullet}\left(\widehat{\mathbf{W}}_{N}\right)$ is a $\mathcal{H o l i e b}{ }^{\diamond}$-algebra equipped with quite explicit strongly homotopy operations. There is a canonical (homogeneous of homological degree zero) injection

$$
u: \quad \begin{array}{ccc}
C y c^{\bullet}\left(\mathbf{W}_{N}\right) & C y c \cdot\left(\widehat{\mathbf{W}}_{N}\right) \\
\left(e_{\alpha_{1}}\left[-p_{1}\right] \otimes \ldots \otimes e_{\alpha_{n}}\left[-p_{n}\right]\right)^{\mathbb{Z}_{n}} & \longrightarrow & u\left(e_{\alpha_{1}}\left[-p_{1}\right] \otimes \ldots \otimes e_{\alpha_{n}}\left[-p_{n}\right]\right)^{\mathbb{Z}_{n}}
\end{array}
$$

identifying each letter $e_{\alpha}[-p]$ in a cyclic word from $C y c\left(\mathbf{W}_{N}\right)$ with a (totally ordered) word in $p+2$ letters

$$
e_{\alpha}[-p] \longrightarrow e_{\alpha}^{\left(0_{p}\right)} \otimes e_{\alpha}^{\left(1_{p}\right)} \otimes \ldots \otimes e_{\alpha}^{\left(p+1_{p}\right)}
$$

i.e.

$u\left(e_{\alpha_{1}}\left[-p_{1}\right] \otimes \ldots \otimes e_{\alpha_{n}}\left[-p_{n}\right]\right)^{\mathbb{Z}_{n}}:=\left(e_{\alpha_{1}}^{(0)_{p_{1}}} \otimes e_{\alpha_{1}}^{(1)_{p_{1}}} \otimes \ldots \otimes e_{\alpha_{1}}^{\left(p_{1}+1\right)_{p_{1}}} \otimes \ldots \otimes e_{\alpha_{n}}^{(0)_{p_{n}}} \otimes e_{\alpha_{n}}^{(1)_{p_{n}}} \otimes \ldots \otimes e_{\alpha_{n}}^{\left(p_{n}+1\right)_{p_{n}}}\right)^{\mathbb{Z}_{m}}$

where

$$
m=n\left(p_{1}+\ldots+p_{n}+2 n\right)
$$

A remarkable an almost obvious fact is that the linear subspace

$$
u\left(C y c\left(\widehat{W}_{N}\right)\right) \subset C y c\left(\widehat{\mathbf{W}}_{N}\right)
$$

is closed with respect to all strong homotopy involutive Lie bialgebra operations and hence is itself a $\mathcal{H}_{\text {olieb }}{ }^{\diamond}$ algebra. In this way we induce a $\mathcal{H}$ olieb ${ }^{\diamond}$-algebra structure

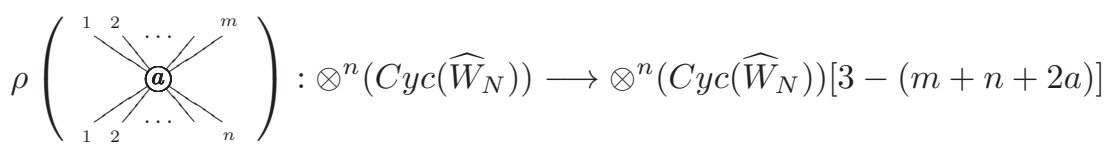

on $C y c\left(\widehat{W}_{N}\right)$. It is immediate to see that all these operations have degree -1 with respect to the weightgrading by the lengths of cyclic words. On the linear subspace

$$
\operatorname{Cyc}\left(W_{N}\right) \subset \operatorname{Cyc}\left(\widehat{W}_{N}\right)
$$

this $\mathcal{H}$ olieb ${ }^{\diamond}$-algebra structure reduces precisely to Schedler's necklace Lie bialgebra structure corresponding to the quiver (11).

\section{A geometric application: a new family of $\mathcal{H}$ olieb $b^{\diamond}$-operations in string topology}

6.1. Poincare duality algebras. Let $A$ be a finite-dimensional differential non-negatively graded unital commutative algebra. We denote the (degree zero) multiplication map in $A$ by center dot,

$$
\begin{array}{ccc}
A \odot A & \longrightarrow & A[0] \\
a \otimes b & \longrightarrow & a \cdot b
\end{array}
$$

A dg algebra $A$ as above is called a $d g$ Poincare duality algebra of degree $n$ if it comes equipped with a degree $-n$ orientation map

$$
\mathfrak{o}: A \rightarrow \mathbb{K}[-n]
$$


such that the induced graded symmetric paring, or scalar product,

$$
\begin{aligned}
\langle,\rangle: A \odot A & \longrightarrow \mathbb{K}[-n] \\
a \odot b & \longrightarrow \mathfrak{o}(a \cdot b)
\end{aligned}
$$

is non-degenerate, and, moreover, $\mathfrak{o}(d a)=0$ for any $a \in A$. This scalar product induces a canonical isomorphism

$$
\imath: A \longrightarrow A^{*}[-n]:=\operatorname{Hom}(A, \mathbb{K})[-n]
$$

such that for any $a, b \in A$ a one has $\imath(a)(b)=\mathfrak{o}(a \cdot b)$. This isomorphism combined with the dualization of the multiplication map induces in turn a degree $n$ diagonal on $A$,

$$
\begin{array}{ccc}
\Delta: A & \longrightarrow & A \odot A[n] \\
a & \longrightarrow & \Delta(a)=: \sum a^{\prime} \otimes a^{\prime \prime}
\end{array}
$$

which satisfies

$$
\Delta(a \cdot b)=\sum\left(a \cdot b^{\prime}\right) \otimes b^{\prime \prime}=\sum(-1)^{|a|\left|b^{\prime}\right|} b^{\prime} \otimes\left(a \cdot b^{\prime \prime}\right)=\sum \sum(-1)^{\left|a^{\prime \prime}\right||b|}\left(a^{\prime} \cdot b\right) \otimes a^{\prime \prime}=\sum a^{\prime} \otimes\left(a^{\prime \prime} \cdot b\right)
$$

for any $a, b \in A$, and hence makes $A$ into a Frobenius algebra. In particular, we have the equalities (presented up to the obvious Koszul sign as in the formulae just above)

$$
\sum \mathfrak{o}\left(a \cdot b \cdot c^{\prime}\right) c^{\prime \prime}= \pm \mathfrak{o}\left(a \cdot b \cdot c^{\prime \prime}\right) c^{\prime}= \pm \mathfrak{o}\left(a^{\prime} \cdot b \cdot c\right) a^{\prime \prime}= \pm \mathfrak{o}\left(a^{\prime \prime} \cdot b \cdot c\right) a^{\prime}= \pm \mathfrak{o}\left(a \cdot b^{\prime} \cdot c\right) b^{\prime \prime}= \pm \mathfrak{o}\left(a \cdot b^{\prime \prime} \cdot c\right) b^{\prime} .
$$

for any $a, b, c \in A$, which we shall use later.

We assume from now on that $A$ is connected and augmented,

$$
A=\mathbb{K} \oplus \bar{A}
$$

with $\bar{A}$ being positively graded. In this case the composite map

$$
\diamond: A \stackrel{\Delta}{\longrightarrow} A \otimes A[n] \stackrel{\cdot}{\longrightarrow} A[n]
$$

vanishes when restricted to the subspace $\bar{A}$.

As $A$ is finite-dimensional, all the above properties (with appropriate degree changes) hold true for its dual $A^{*}$, i.e. $A^{*}$ is also Frobenius algebra but with the diagonal in degree zero and the multiplication map in degree $+n$.

Given a compact $n$-dimensional manifold $M$, a Poincare model for $M$ is, by definition, a dg Poincare duality algebra $A$ of degree $n$ which is quasi-isomorphic (as an $\mathcal{A} s s_{\infty}$ algebra) to the de Rham algebra $\Omega_{M}^{\bullet}$ of differential forms on $M$. It was proven in [LS that every connected and simply connected manifold $M$ admits a Poincare model.

6.2. Cyclic Hochschild complexes and equivariant (co)homology of free loop spaces. Recall (see $\$ 2.5$ or Corollary 6.3.1) that any linear map of the form

$$
\Theta_{2}: \otimes^{2}(W[d])_{\mathbb{Z}_{2}} \longrightarrow \mathbb{K}[1+d], \quad d \in \mathbb{Z},
$$

makes the (reduced) space of cyclic words in $W$

$$
C y c(W)=\oplus_{k \geq 0}\left(\otimes^{k} W\right)_{\mathbb{Z}_{k}} \text { or } \overline{C y c}(W)=\oplus_{k \geq 1}\left(\otimes^{k} W\right)_{\mathbb{Z}_{k}},
$$

into a $\mathcal{L} i e b_{d}^{\diamond}$-algebra. Let us apply this observation to an arbitrary Poincare duality algebra $A$ in degree $n$. Note that the scalar product (16) induces a non-degenerate graded symmetric linear map,

$$
\begin{aligned}
& \Theta_{2}^{\prime}: \quad \otimes^{2}(A[n])_{\mathbb{Z}_{2}} \quad \longrightarrow \quad \mathbb{K}[n] \\
& \mathfrak{s}^{n} a \otimes \mathfrak{s}^{n} b \quad \longrightarrow \mathfrak{s}^{n} O(a \cdot b)
\end{aligned}
$$


Indeed, its graded commutativity (i.e. invariance under $\mathbb{Z}_{2} \simeq \mathbb{S}_{2}$ ) can be checked directly,

$$
\begin{aligned}
\Theta_{2}^{\prime}\left(\mathfrak{s}^{n} b \otimes \mathfrak{s}^{n} a\right) & =(-1)^{|a||b|} \mathfrak{s}^{n} o(a \cdot b) \\
& =(-1)^{\left(\left|\mathfrak{s}^{n} a\right|+n\right)\left(\left|\mathfrak{s}^{n} b\right|+n\right)} \mathfrak{s}^{n} o(a \cdot b) \\
& =(-1)^{n^{2}+n\left(\left|\mathfrak{s}^{n} \alpha\right|+\left|\mathfrak{s}^{n} b\right|\right)+\left|\mathfrak{s}^{n} \alpha\right| \cdot\left|\mathfrak{s}^{n} \beta\right|} \Theta_{2}\left(\mathfrak{s}^{n} a \otimes \mathfrak{s}^{n} b\right) \\
& =(-1)^{n^{2}-n^{2}+\left|\mathfrak{s}^{n} a\right| \cdot\left|\mathfrak{s}^{n} b\right|} \Theta_{2}\left(\mathfrak{s}^{n} a \otimes \mathfrak{s}^{n} b\right) \\
& =(-1)^{\left|\mathfrak{s}^{n} \alpha\right| \cdot\left|\mathfrak{s}^{n} b\right|} \Theta_{2}^{\prime}\left(\mathfrak{s}^{n} a \otimes \mathfrak{s}^{n} b\right)
\end{aligned}
$$

for any $a, b \in A$. Here we used the fact that both sides are zero unless $\left|\mathfrak{s}^{n} a\right|+\left|\mathfrak{s}^{n} b\right|=-n$. Setting $d:=n-1$ we rewrite the map $\Theta_{2}^{\prime}$ as

$$
\Theta_{2}^{\prime}: \otimes^{2}((A[1])[d]) \longrightarrow \mathbb{K}[d+1]
$$

and conclude that the spaces of cyclic words $C y c(A[1])$ and (hence) $\overline{C y c}(\bar{A}[1])$ come equipped with a $\mathcal{L}_{i e b_{n-1^{-}}}$ structure, that is, with compatible Lie brackets $\{$,$\} and coproduct \Delta$, both operations having degree $1-d=2-n$.

The scalar product (16) induces also a non-degenerate linear map,

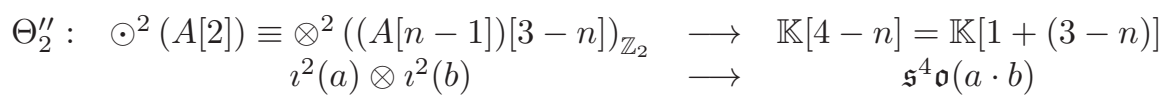

implying — using the above general construction for $d=3-n$ - that the vector spaces

$$
C y c(A[n-1]) \simeq C y c\left(A^{*}[-1]\right) \text { and } \overline{C y c}\left(\bar{A}^{*}[-1]\right)
$$

come equipped with an induced $\mathcal{L} i e b_{3-n}^{\diamond}$-algebra structures, with both basic operations, $\{$,$\} and \Delta$, having degree $1-(3-n)=n-2$. Here we used the canonical isomorphism $A[n]=A^{*}$. It is precisely the dual (in the obvious sense)) of the $\mathcal{L} i e b_{n-2}^{\diamond}$-algebra structure on $C y c(A[1])$ and, respectively, $\overline{C y c}(\bar{A}[1])$ discussed just above.

6.2.1. Hochschild differential. The dual of the multiplication map $A \otimes A \rightarrow A$

$$
\begin{aligned}
\triangle: A^{*} & \longrightarrow A^{*} \otimes A^{*} \\
a & \longrightarrow \sum a^{\prime} \otimes a^{\prime \prime}
\end{aligned}
$$

makes the vector space $C y c\left(A^{*}[-1]\right)$ into a complex. If we represents pictorially an element $\left(\mathfrak{s}^{-1} a_{1} \otimes \mathfrak{s}^{-1} a_{2} \otimes\right.$ $\left.\ldots \otimes \mathfrak{s}^{-1} a_{i} \otimes \ldots \otimes \mathfrak{s}^{-1} a_{n}\right) \in C y c\left(A^{*}[-1]\right)$ as a circle-like graph with $n$ vertices,

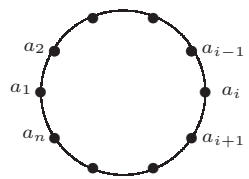

with $n$ vertices decorated by elements of $A^{*}[-1]$ and $n$ edges, then the Hochschild differential $d_{H}$ in $\operatorname{Cyc}\left(A^{*}[-1]\right)$ is defined as an alternating sum over splitting each vertex into two new vertices as explained pictorially below,

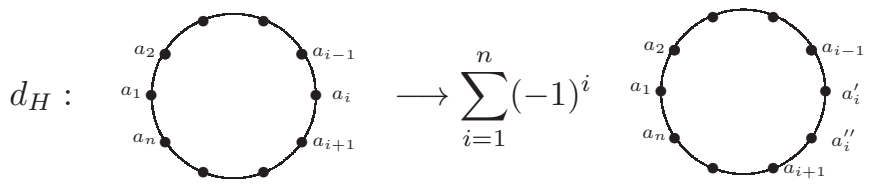

Similarly, the reduced diagonal

$$
\bar{\triangle}: \bar{A}^{*} \longrightarrow A^{*} \stackrel{\triangle}{\longrightarrow} A^{*} \otimes A^{*} \longrightarrow \bar{A}^{*} \otimes \bar{A}^{*}
$$

makes the grade vector space $\left(\overline{C y c}\left(\bar{A}^{*}[-1]\right)\right)$ into a complex which sometimes has a nice geometric interpretation (see the next subsection).

It was shown in [CEG] (see also [CFL, $\mathrm{NW}$ for alternative proofs) that the above mentioned $\mathcal{L} i e b_{3-n^{-}}^{\diamond}$ algebra structure on $\left(\overline{C y c}\left(\bar{A}^{*}[-1]\right)\right)$ is respected by the differential $d_{H}$ and hence survives at the cohomology level; 
we conclude that $H^{\bullet}\left(\left(\overline{C y c}\left(\bar{A}^{*}[-1]\right)\right), d_{H}\right)$ comes equipped with compatible Lie bracket and Lie cobracket of degree $n-2$ for any Poincare duality algebra $A$ in degree $n$. Similarly, one gets a canonical $\mathcal{L}_{i e b_{n-1}}^{\diamond}$ algebra structure on $H^{\bullet}\left((\overline{C y c}(\bar{A}[1])), d_{H}^{*}\right)$ which has been much studied in $[\mathrm{NW}$ (with many nice explicit formulae obtained).

6.2.2. $\mathcal{L} i e b^{\diamond}$ structures on equivariant cohomology of free loop spaces. Let $L M$ stand for the space of free loops in a closed oriented $n$-dimensional manifold $M$, and $\bar{C}_{S^{1}}^{\bullet}(L M)\left(\right.$ resp., $\left.\bar{C}^{S^{1}}(L M)\right)$ for the reduced equivariant cochain (resp., chain) complex of $L M$ with respect to the obvious $S^{1}$ action on $L M$; note that we assume that $\bar{C}_{\bullet}^{S^{1}}(L M)$ is the genuine linear dual of $\bar{C}_{S^{1}}^{\bullet}(L M)$ as a $\mathbb{Z}$-graded complex, i.e. it is negatively graded and hence has the boundary differential of cohomological degree +1 . Let $\bar{H}_{S^{1}}^{\bullet}(L M)\left(\operatorname{resp} ., \bar{H}_{\bullet}^{S^{1}}(L M)\right)$ stand for its cohomology.

Assume $A$ is a Poincare model of $M$, then there are canonical morphisms of cohomology groups

$$
\bar{H}_{\bullet}^{S^{1}}(L M) \longrightarrow H^{\bullet}\left(\overline{C y c}\left(\bar{A}^{*}[-1]\right), d_{H}\right), \quad H^{\bullet}\left(\overline{C y c}(\bar{A}[1]), d_{H}^{*}\right) \longrightarrow \bar{H}_{S^{1}}^{\bullet}(L M)
$$

which are isomorphisms if $M$ is a closed connected and simply connected manifold. In this case both the equivariant (co)homology groups $H_{\bullet}^{S^{1}}(L M)$ and $H_{S^{1}}^{\bullet}(L M)$ come equipped with induced involutive Lie bialgebra structure which has been first discovered - in a very nice geometric way - by M. Chas and D. Sullivan in $\mathrm{CS}$.

6.3. A new class of $\mathcal{H}$ olie $b_{d}^{\diamond}$-algebra structures from Poincare duality algebras and props of ribbon hypergraphs. Our main result in this paper is the observation that for any a graded vector space $W$ equipped with a linear map

$$
\Theta_{p}:(W[d])_{\mathbb{Z}_{p}}^{\otimes p} \rightarrow \mathbb{K}[1+d]
$$

for some $d \in \mathbb{Z}$ and $p \geq 2$, the associated vector spaces of "cyclic words in $W$ ",

$$
C y c(W):=\bigoplus_{n \geq 0}\left(W^{\otimes n}\right)^{\mathbb{Z}_{n}}, \quad \overline{C y c}(W):=\bigoplus_{n \geq 1}\left(W^{\otimes n}\right)^{\mathbb{Z}_{n}}
$$

come equipped canonically with a $\mathcal{H}$ olieb $_{d}^{\diamond}$-algebra structure given by explicit formulae (14). Given a Poincare duality algebra $A$ in degree $n$ and the associated linear maps

$$
\begin{aligned}
& \mathfrak{o}_{p}: \quad \odot^{p} A \quad \longrightarrow \quad \mathbb{K}[-n] \\
& \alpha_{1} \otimes \cdots \otimes \alpha_{p} \longrightarrow \mathfrak{o}\left(\alpha_{1} \cdots \cdots \alpha_{p}\right),
\end{aligned}
$$

can we use the above construction to induce a $\mathcal{H}_{\text {olieb }}{ }_{d}^{\text {-algebra structure on } C y c(A[1]) \text { or } C y c\left(A^{*}[-1]\right) \text { for }}$ suitable $d, p \in \mathbb{Z}$ ?

CASE 1. Consider first the case $W=A[1]$. Out of the map $\mathfrak{o}_{p}$ we would like to built a map

$$
\Theta_{p}:(W[d])_{\mathbb{Z}_{p}}^{\otimes p} \rightarrow \mathbb{K}[1+d] .
$$

We can rewrite (19) as

$$
\mathfrak{o}_{p}: \odot^{p}(W[-1]) \longrightarrow \mathbb{K}[-n]
$$

or equivalently as

$$
\mathfrak{o}_{p}: \otimes^{p}(W[-1+m]) \longrightarrow \mathbb{K}[-n+m p], \quad \forall m \in \mathbb{Z} .
$$

The equations

$$
d=-1+m, \quad 1+d=-n+m p
$$

imply

$$
n=m(p-1)
$$

Hence the only solution which applies to every Poincare duality algebra (even to the one whose degree is a prime number) is $p=2$ in which case $n=m, d=n-1$; hence in this case we recover the Chas-Sullivan $\mathcal{L} i e b_{n-1}^{\diamond}$-algebra structure on $C y c(A[1])$ discussed above.

CASE 1. Consider next the case $W=A[n-1] \simeq A^{*}[-1]$. Then we can rewrite (19) as

$$
\mathfrak{o}_{p}: \odot^{p}(W[1-n]) \longrightarrow \mathbb{K}[-n]
$$


or equivalently as

The equations

$$
\mathfrak{o}_{p}: \otimes^{p}(W[1-n+m]) \longrightarrow \mathbb{K}[-n+m p], \quad \forall m \in \mathbb{Z}
$$

$$
d=1-n+m, \quad 1+d=-n+m p
$$

imply

$$
2=m(p-1)
$$

Here we get precisely two solutions. The first solution is $p=2$ implying $m=2, d=3-n$; hence in this case we recover the Chas-Sullivan $\mathcal{L} i e b_{3-n}^{\diamond}$-algebra structure on $\operatorname{Cyc}\left(A^{*}[-1]\right)$ discussed above. The second solution is $p=3, m=1, d=2-n$ which is studied in the following

6.3.1. Proposition. Given any dg connected Poincare duality algebra $A$ of degree $n$. The graded vector space $\overline{C y c}\left(\bar{A}^{*}[-1]\right)$ comes equipped canonically with a Holieb ${ }_{2-n}^{\diamond}$-algebra structure induced by the map

$$
\begin{array}{cccc}
\mathfrak{o}_{3}: & \odot^{3} A & \longrightarrow & \mathbb{K}[-n] \\
& a \odot b \odot c & \longrightarrow & \mathfrak{o}(a \cdot b \cdot c),
\end{array}
$$

whose only possibly non-trivial operations are controlled by the following four generators of $\mathcal{H}$ olie $b_{2-n}^{\diamond}$,

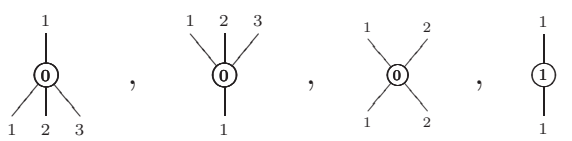

which in turn are controlled via the canonical morphism (14) by the following (respectively) ribbon hypergraphs from $\mathcal{R} \mathcal{H} \mathrm{ra}_{2-n}$,

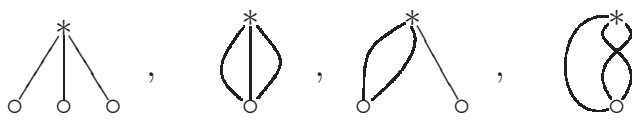

with vertices and boundaries appropriately (skew)symmetrized (so that their labelling is omitted).

Moreover, these four operations respect the Hochschild differential $d_{H}$ in $\overline{C y c}\left(\bar{A}^{*}[-1]\right)$ and hence induce a $\mathcal{H}$ olieb ${ }_{2-n}$-algebra structure on the cohomology $H^{\bullet}\left(\overline{C y c}\left(\bar{A}^{*}[-1]\right), d_{H}\right)$.

Proof. We have to prove only the last statement in the above Proposition as the rest is simply a direct application of Corollary to the case $p=3$ and $W=\bar{A}^{*}[-1]$.

Let us first study an action of the graph $\Gamma_{1}:=\bigwedge_{0}$ on $\overline{C y c}\left(\bar{A}^{*}[-1]\right)$ via the canonical representation $\rho_{\Theta_{3}}$ introduced in the proof of Theorem 3.5.1 for the particular case when $W=\bar{A}^{*}[-1]$. Up to the standard Koszul signs this action,

$$
\rho_{\Theta_{3}}\left(\Gamma_{1}\right): \overline{C y c}\left(\bar{A}^{*}[-1]\right) \bigotimes \overline{C y c}\left(\bar{A}^{*}[-1]\right) \bigotimes \overline{C y c}\left(\bar{A}^{*}[-1]\right) \longrightarrow \overline{C y c}\left(\bar{A}^{*}[-1]\right)[2 n-3]
$$

is given by the following formula (cf.

$$
\begin{aligned}
\rho_{\Theta_{3}}\left(\Gamma_{1}\right):\left(a_{1} \otimes \ldots \otimes a_{p}\right)_{\mathbb{Z}_{p}} \otimes\left(b_{1} \otimes \ldots \otimes b_{q}\right)_{\mathbb{Z}_{q}} \otimes\left(c_{1} \otimes \ldots \otimes c_{r}\right)_{\mathbb{Z}_{r}} \longrightarrow \sum_{i=1}^{p} \sum_{j=1}^{p} \sum_{k=1}^{r} \\
\quad \pm \mathfrak{o}\left(\mathfrak{s}^{1-n} a_{i} \cdot \mathfrak{s}^{1-n} b_{j} \cdot \mathfrak{s}^{1-n} c_{k}\right)\left(a_{i+1} \otimes \ldots \otimes a_{i-1} \otimes c_{k+1} \otimes \ldots \otimes c_{k-1} \otimes b_{j+1} \otimes \ldots \otimes b_{j-1}\right)^{\mathbb{Z}_{N}}
\end{aligned}
$$

where we used the isomorphism $A^{*}[-1] \simeq A[n-1]$ and set $N=p+q+r-3$. The Hochschild differential splits inputs as follows

$$
a_{i} \rightarrow \sum a_{i}^{\prime} \otimes a_{i}^{\prime \prime}, \quad b_{j} \rightarrow \sum b_{j}^{\prime} \otimes b_{j}^{\prime \prime}, \quad c_{k} \rightarrow \sum c_{k}^{\prime} \otimes c_{k}^{\prime \prime} .
$$

Hence the obstructions for the above map to respect the Hochschild differential $d_{H}$ are given by the summands of the form,

$$
\begin{aligned}
& \mathfrak{o}\left(\mathfrak{s}^{1-n} a_{i}^{\prime} \cdot \mathfrak{s}^{1-n} b_{j} \cdot \mathfrak{s}^{1-n} c_{k}\right)\left(\ldots \otimes b_{j-1} \otimes a_{i}^{\prime \prime} \otimes a_{i+1} \otimes \ldots\right)+\mathfrak{o}\left(\mathfrak{s}^{1-n} a_{i}^{\prime \prime} \cdot \mathfrak{s}^{1-n} b_{j} \cdot \mathfrak{s}^{1-n} c_{k}\right)\left(\ldots \otimes a_{i-1} \otimes a_{i}^{\prime \prime} \otimes c_{k+1} \otimes \ldots\right)+ \\
& \pm \mathfrak{o}\left(\mathfrak{s}^{1-n} a_{i} \cdot \mathfrak{s}^{1-n} b_{j}^{\prime} \cdot \mathfrak{s}^{1-n} c_{k}\right)\left(\ldots \otimes c_{k-1} \otimes b_{j}^{\prime \prime} \otimes b_{j+1} \otimes \ldots\right)+\mathfrak{o}\left(\mathfrak{s}^{1-n} a_{i} \cdot \mathfrak{s}^{1-n} b_{j}^{\prime \prime} \cdot \mathfrak{s}^{1-n} c_{k}\right)\left(\ldots \otimes b_{j-1} \otimes b_{j}^{\prime} \otimes a_{i+1} \otimes \ldots\right) \\
& \pm \mathfrak{o}\left(\mathfrak{s}^{1-n} a_{i} \cdot \mathfrak{s}^{1-n} b_{j} \cdot \mathfrak{s}^{1-n} c_{k}^{\prime}\right)\left(\ldots \otimes c_{k-1} \otimes c_{k}^{\prime \prime} \otimes b_{j+1} \otimes \ldots\right)+\mathfrak{o}\left(\mathfrak{s}^{1-n} a_{i} \cdot \mathfrak{s}^{1-n} b_{j} \cdot \mathfrak{s}^{1-n} c_{k}^{\prime \prime}\right)\left(\ldots \otimes a_{i-1} \otimes c_{k}^{\prime} \otimes c_{k+1} \otimes \ldots\right)
\end{aligned}
$$


in which precisely one of the "dashed" elements lands inside the orientation map o. Thanks to identity (17), all these terms come in pairs which cancel each other (for example, the first term in the first row cancels the second term in the second row and so on).

Consider next the operation on $\overline{C y c}\left(\bar{A}^{*}[-1]\right)$ controlled by the graph $\Gamma_{2}=\left(\bigcup_{0}\right.$. The obstructions to this operations under the canonical morphism $\rho_{\Theta_{3}}\left(\Gamma_{2}\right)$ to respect the Hochschild differential $d_{H}$ are given by the terms of two types,

(i) one type corresponds to summands in which precisely one of the "dashed" elements lands inside the orientation map as above; these terms cancel each other due to the same identities (17) as above;

(ii) the second type corresponds to summands in which both "dashed" elements lands inside the orientation map, i.e. to summands which contain a numerical factor of the form

$$
\mathfrak{o}\left(\mathfrak{s}^{1-n} a_{i}^{\prime} \cdot \mathfrak{s}^{1-n} a_{i}^{\prime \prime} \cdot \mathfrak{s}^{1-n} b\right)
$$

All these summands in (ii) vanish dues to the fact that the map (18) vanishes on elements of $\overline{C y c}\left(\bar{A}^{*}[-1]\right)$. The obstructions for the two remaining operations,

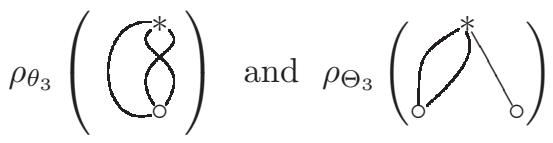

to respect $d_{H}$ are of the two types discussed above; they vanish for the same reasons.

6.3.2. Corollary. For any closed connected and simply connected $n$-dimensional manifold $M$ the reduced

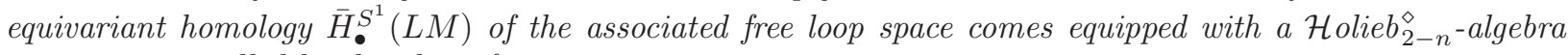
structure controlled by the above four operations.

It is easy to see that these four new $\mathcal{H} o l i e b_{2-n}^{\diamond}$ operations on $\bar{H}_{\bullet}^{S^{1}}(L M)$ are non-trivial for $M=\mathbb{C P}^{n}$ with $n \geq 3$.

\section{REFERENCES}

[AKKN1] A. Alekseev, N. Kawazumi, Y. Kuno and F. Naef, The Goldman-Turaev Lie bialgebra in genus zero and the Kashiwara-Vergne problem, Adv. Math. 326 (2018) 1-53

[AKKN2] A. Alekseev, N. Kawazumi, Y. Kuno and F. Naef, The Goldman-Turaev Lie bialgebra and the Kashiwara-Vergne problem in higher genera, arxiv arXiv:1703.0581

[AN] A. Alekseev and F. Naef, Goldman-Turaev formality from the Knizhnik-Zamolodchikov connection, C. R. Math. Acad. Sci. Paris 355 (2017), no. 11, 1138-1147.

[Ba1] S. Barannikov, Noncommutative Batalin-Vilkovisky geometry and matrix integrals. C. R. Math. Acad. Sci. Paris 348 (2010), no. 7-8, 359-362

[Ba2] S. Barannikov, Matrix De Rham complex and quantum A-infinity algebras, Lett. Math. Phys. 104 (2014) $373-395$.

[CS] M. Chas and D. Sullivan, Closed string operators in topology leading to Lie bialgebras and higher string algebra, arXiv:math/0212358

[CMW] R. Campos, S. Merkulov and T. Willwacher The Frobenius properad is Koszul, Duke Math. J. 165, No.1 (2016), 2921-2989.

[Ch] M. Chas, Combinatorial Lie bialgebras of curves on surfaces, Topology 43 (2004), no. 3, 543-568.

[CEG] X. Chen, F. Eshmatov, and W. L. Gan, Quantization of the Lie bialgebra of string topology, Comm. Math. Phys. 301(1) (2011) 37-53.

[CFL] K. Cieliebak, K. Fukaya and J. Latschev, Homological algebra related to surfaces with boundary, preprint arXiv:1508.02741, 2015.

[CS] M. Chas and D. Sullivan, Closed string operators in topology leading to Lie bialgebras and higher string algebra, in: The legacy of Niels Henrik Abel, pp. 771-784, Springer, Berlin, 2004.

[D1] V. Drinfeld, Hamiltonian structures on Lie groups, Lie bialgebras and the geometric meaning of the classical Yang-Baxter equations, Soviet Math. Dokl. 27 (1983) 68-71.

[D2] V. Drinfeld, On quasitriangular quasi-Hopf algebras and a group closely connected with Gal $(\bar{Q} / Q)$, Leningrad Math. J. 2, No. 4 (1991), 829-860.

[DCTT] G. C. Drummond-Cole, J. Terilla and T. Tradler, Algebras over Cobar(coFrob), J. Homotopy Relat. Struct. 5 (2010), no.1, 15-36.

[ES] P. Etingof and O. Schiffmann, Lectures on Quantum Groups, International Press, 2002. 
[G] W. Goldman, Invariant functions on Lie groups and Hamil- tonian flows of surface group representations, Invent. Math. 85 (1986), no. 2, 263-302.

[Kr] O. Kravchenko, Deformations of Batalin-Vilkovisky algebras, In: Poisson geometry (Warsaw, 1998), Banach Center Publ., vol. 51, Polish Acad. Sci., Warsaw, 2000, pp. 131-139.

[Ku] Yusuke Kuno, private communication, December 2016.

[LS] P. Lambrechts and D. Stanley, Poincare duality and commutative differential graded algebras, Ann. Sci. Ec. Norm. Super. 41(4) (2008) 495-509.

[LZ] S.K. Lando and A.K. Zvonkin, Graphs on Surfaces and their Applications, Springer, 2004

[MaVo] M. Markl and A.A. Voronov, PROPped up graph cohomology, in: Algebra, arithmetic, and geometry: in honor of Yu. I. Manin. Vol. II, Progr. Math., 270, Birkhäuser Boston, Inc., Boston, MA (2009) pp. 249-281.

[Ma] G. Massuyeau, Formal descriptions of Turaev's loop operations, Quantum Topol. 9 (2018) 39-117.

[MV] S. Merkulov and B. Vallette, Deformation theory of representations of prop(erad)s I 8 II, Journal für die reine und angewandte Mathematik (Qrelle) 634, 51-106, \& 636, 123-174 (2009)

[MW1] S. Merkulov and T. Willwacher, Props of ribbon graphs, involutive Lie bialgebras and moduli spaces of curves, preprint arXiv:1511.07808 (2015) 51pp.

[MW2] S.A. Merkulov and T. Willwacher, Classification of universal formality maps for quantizations of Lie bialgebras, Compositio Mathematica, 156 (2020) 2111-2148

[NW] F. Naef and T. Willwacher, String topology and configuration spaces of two points, preprint arXiv:1911.06202 (2019).

[P] R.C. Penner, Perturbative series and the moduli space of Riemann surfaces, J. Differential Geometry 27 (1988), no. 1, $35-53$

[S] T. Schedler, A Hopf algebra quantizing a necklace Lie algebra canonically associated to a quiver. Intern. Math. Res. Notices (2005), 725-760.

[T] V.G. Turaev, Skein quantization of Poisson algebras of loops on surfaces, Ann. Sci. Ecole Norm. Sup. (4) 24, no. 6, (1991) 635-704.

[TZ] T. Tradler and M. Zeinalian, Algebraic string operations, K-Theory 38 (2007), no. 1, 59 - 82.

[V] B. Vallette, A Koszul duality for props, Trans. Amer. Math. Soc., 359 (2007), 4865-4943.

Sergei Merkulov: Mathematics Research Unit, Luxembourg University, Grand Duchy of Luxembourg

Email address: sergei.merkulov@uni.lu 